\title{
Local and nonlocal continuum modeling of inelastic periodic networks applied to stretching-dominated trusses
}

\author{
A. Desmoulins ${ }^{\mathrm{a}}$, D. M. Kochmann ${ }^{\mathrm{b}, *}$ \\ ${ }^{a}$ Département de Mécanique, Ecole Polytechnique, Palaiseau 91128, France \\ ${ }^{b}$ Graduate Aerospace Laboratories, California Institute of Technology, Pasadena, CA 91125, U.S.A.
}

\begin{abstract}
We present a nonlocal continuum model and its numerical implementation to describe the macroscale response of periodic discrete networks via second-order homogenization. The scale-bridging technique is applied to the specific example of stretching-dominated elastic and inelastic periodic truss networks. Experiments on small-scale truss structures have highlighted the importance of nodal connections on the effective stiffness and strength. Therefore, we describe the mechanics of trusses by accounting for the stretching of truss members and the deformation of nodes. For the representative 2D examples of lattices having square and triangular architectures and for example bar and nodal constitutive laws, we show that a simple continuum model based on affinely deforming a representative unit cell is sufficient to reproduce the nonlinear elastic behavior of discrete trusses. By contrast, localization that arises, e.g., from inelastic deformation requires a refined model. This is where the presented nonlocal continuum model is capable of accurately capturing details of localized deformation. We illustrate the performance of the model by comparing the results of example finite element simulations using the continuum constitutive model to discrete lattice calculations with elastic-plastic bars. Optimal performance is achieved when the representative unit cell of the continuum model agrees with the actual size of the discrete truss unit cell, which accounts for size effects even in regimes where a separation of scales between finite element size and unit cell size does not strictly apply.
\end{abstract}

Keywords: nonlocal model, elasticity, plasticity, homogenization, truss

\section{Introduction}

Over the past decade, the advent of micro- and nanoscale additive manufacturing techniques has revived the interest in accurately predicting the mechanics of truss structures across scales. Trusses have served as building blocks for hierarchical and periodic (meta-)materials with interesting effective properties resulting form the collective response of structural members across multiple scales. Examples include microtrusses (Schaedler et al., 2011a; Torrents et al., 2012) and nanotrusses (Meza et al., 2014), which can be tailored, e.g., for optimal stiffness and strength (Deshpande et al., 2001a; Meza et al., 2015), nonlinear stress-strain behavior (Fleck, 2001; Fan et al., 2009), fracture toughness (Romijn and Fleck, 2007; Fleck et al., 2010b), energy absorption (Wadley et al., 2008; Kumar, 2011), or acoustic wave propagation (Ruzzene and Scarpa, 2005; Gonella and Ruzzene, 2008; Krödel et al., 2014). Especially in the nonlinear elastic regime, the observed wide mechanical hysteresis (associated with plasticity or buckling of structural members) furnishes truss networks with high energy absorption and accommodates large reversible deformation at the nanoscale (Schaedler et al., 2011b; Meza et al., 2014). The mechanical performance of such architected materials is a function of the deformation mechanism, the relative density, and the constituent material properties, see, e.g., Fleck et al. (2010a); Hutchinson and Fleck (2006); Symons and Fleck (2008); Fleck and Qiu (2007); Jacobsen et al. (2007); Zheng et al. (2014); Valdevit et al. (2013) for recent studies.

The new opportunities to create such versatile materials systems also call for advanced modeling techniques, in particular since the number of individual truss members in micro- and nanotrusses can increase dramatically compared

*Phone +1-626-395-8113, fax +1-626-395-2900.

Email address: kochmann@caltech.edu (D. M. Kochmann) 
to macroscopic truss structures. Modeling the mechanical response of trusses composed of thousands to millions of beams becomes computationally costly, especially if those can no longer be modeled as simple elastic-plastic axial bars or Bernoulli or Timoshenko beams but require refined models; see, e.g., the recent investigation of hollow ceramic nanotrusses by Meza et al. (2014, 2015); Montemayor (2015) which demonstrate a vastly different behavior than their micro- or macroscopic counterparts. In such scenarios, full-resolution finite element (FE) simulations cease to be feasible and alternative strategies are required to describe the effective response of complex truss networks, once the behavior of individual truss members is known. This defines a general scale-bridging challenge to be addressed here: given the constitutive description of discrete lattice members, how can the effective response be described accurately by a continuum model that qualifies to replace the costly discrete calculation? In fact, this question goes beyond truss networks and generally applies to discrete periodic networks including, e.g., atomistic crystals, and granular media.

A large amount of previous work has been devoted to the theoretical and computational modeling and optimization of truss lattices, see e.g. Fleck (2001); Deshpande et al. (2001a); Queheillalt and Wadley (2005); Wadley (2006); Wadley et al. (2008); Fan et al. (2009); Kumar (2011); Valdevit et al. (2013). A prominent strategy has been to introduce effective continuum models that approximate the discrete truss behavior in an efficient manner. A variety of such continuum models have been developed, e.g., for linear elastic beam and plate-like truss structures (Noor and Andersen, 1979; McCallen and Romstad, 1988; Necib and Sun, 1989; McCallen and Romstad, 1990; Wu and Chen, 1994; Usik, 1994). At the core of these techniques stands the identification of a representative unit cell (RUC) and the derivation of the effective continuum quantities such as energy density, stresses and incremental stiffness tensors from an appropriate deformation of the unit cell (e.g., in an affine manner or by enforcing periodicity). Alternatively, the equivalence between discrete and continuum models can be enforced by equating their eigenfrequencies. However, this method (and all of the above) assumes linearized kinematics; see also the more recent studies of Gonella and Ruzzene (2008) and Zhang et al. (2010).

In finite deformations, more complex phenomena including instability and localization gain importance. To capture those phenomena, refined models have incorporated higher-order gradient terms in the description of the unit cell deformation (using the traditional or extended Cauchy-Born rule), see e.g. Triantafyllidis and Bardenhagen (1993); Bardenhagen and Triantafyllidis (1994) and more recently Askes and Metrikine (2005); Metrikine and Askes (2006), following the concepts of Mindlin (1965). For elastic trusses, this results in gradient elasticity theories (Toupin, 1962, 1964; Koiter, 1964) whose second-order deformation gradients and couple stresses necessitate a special numerical treatment, see e.g. Papanicolopulos et al. (2009); Rudraraju et al. (2014). In a similar spirit, micropolar continuum theories (Dow and Huyer, 1989; De Borst et al., 1993; Kumar and McDowell, 2004) have introduced rotational degrees of freedom and associated couple stresses, inspired by the early theories of Eringen (1965, 1966). Further related approaches for truss networks include those of Wang and Cuitiño (2000); Odegard et al. (2002); Hu and Park (2013). We note that similar continuum models have been developed for other discrete mechanical systems such as graphene sheets (Reddy et al., 2005; Shokrieh and Rafiee, 2010) or carbon nanotubes (Sun and Liew, 2008). All of the above continuum representations assume a separation of scales and fail during localization or failure. In order to retain full accuracy in such regions, the quasicontinuum (QC) method (Tadmor et al., 1999) has been adapted to produce continuum truss models. While originally introduced to coarse-grain atomic crystals (Tadmor et al., 1996; Amelang et al., 2015), recent QC extensions have been developed to bridge the scales in trusses from full resolution to a continuum description by coarsening the discrete set of nodes in truss networks (Beex et al., 2014; Kochmann and Amelang, 2016). This provides full accuracy in regions of interest while efficiently treating the remainder of the simulation domain by an effective continuum model of the above type (commonly employing the Cauchy Born rule). Yet, it also requires a complex simulation setup with well-matched discrete and continuum regions.

Our goal is a continuum model that accurately reflects nonlinear and inelastic deformation mechanisms, and we aim for numerical simplicity by avoiding additional field variables such as rotational degrees of freedom in micropolar theories. In principle, bending of trusses can be described in two ways: by introducing translational and rotational degrees of freedom (such as in classical Euler-Bernoulli beam theory) or by using translational degrees of freedom from which angle changes can be derived (Wang and Cuitiño, 2000) ${ }^{1}$. The former has the advantage of admitting simple beam descriptions at the unit cell level while being computationally more involved; the latter is computationally simple, as shown here, but requires a translation-based description of beam bending. Here, we formulate a general

\footnotetext{
${ }^{1}$ See also the homogenization approach of Pal et al. (2016), which appeared while this contribution was under review.
} 
micro-to-macro transition scheme that applies to, in principle, any discrete network whose constitutive formulation depend on translational degrees of freedom only. As a simple test scenario, we apply the scheme to stretchingdominated (i.e., high connectivity) truss lattices including inelasticity and localization but excluding beam buckling. Our study is further guided by nanoscale experiments on hollow truss lattices, which emphasized the enormous impact of the (hollow) nodes on deformation and failure mechanisms (Meza et al., 2015) - a fact that is often neglected by focusing on stretching or bending of truss members as the only mechanism to store elastic energy. The above nonlocal continuum models (although restricted to elastic trusses) provide the basis for extensions to inelasticity, such as the one presented here.

Our nonlocal continuum model derives from a micro-to-macro transition scheme that applies the extended CauchyBorn rule to a RUC, whose deformation is characterized by independent constitutive models for bars and nodes, allowing for inelastic descriptions such as plasticity and failure. Our numerical implementation is based on a discretization of the nonlocal Galerkin weak form using $C^{1}$-basis functions, and it is applied to representative two-dimensional (2D) truss structures. We show that, while a local continuum is sufficient to reproduce smooth elastic behavior, the nonlocal formulation is required to capture localization, e.g., during plastic deformation. Section 2 summarizes the discrete truss model and the micro-to-macro transition scheme along with the resulting continuum model. Section 3 discusses the numerical implementation, followed by numerical examples shown in Section 4.

\section{Discrete and continuum truss models}

\subsection{Discrete truss description}

As shown in Fig. 1b, we consider a periodic network composed of $N_{n}$ nodes whose positions in the deformed and undeformed configuration are denoted by $\boldsymbol{x}_{\alpha}$ and $\boldsymbol{X}_{\alpha}\left(\alpha=1, \ldots, N_{n}\right)$, respectively. Nodal displacements follow as $\boldsymbol{u}_{\alpha}=\boldsymbol{x}_{\alpha}-\boldsymbol{X}_{\alpha}$ (we use the same coordinate system for both configurations). The nodes are connected by $N_{b}$ bars ${ }^{2}$. For a bar connecting nodes $\alpha$ and $\beta, \boldsymbol{l}_{\alpha \beta}=\boldsymbol{x}_{\alpha}-\boldsymbol{x}_{\beta}$ and $\boldsymbol{L}_{\alpha \beta}=\boldsymbol{X}_{\alpha}-\boldsymbol{X}_{\beta}$ denote its end-to-end vectors in the deformed and undeformed configuration, respectively. Note that, here and in the following, we use Greek indices to denote specific nodes and bars so no summations are implied, whereas Latin indices will assume the conventional tensor summation conventions. Further, we denote a unit vector by $\hat{\boldsymbol{l}}=\boldsymbol{l} / l$ with length $l=|\boldsymbol{l}|$. Each bar is defined by a node pair $v=\alpha \beta$ and we denote by $\mathcal{B}$ the set of all $N_{b}$ pairs. The deformed angle $\theta_{\alpha \beta}^{\gamma} \in(-\pi, \pi)$ between two adjacent bars (with shared central node $\gamma$ and the two external nodes $\alpha$ and $\beta$ ) is given by

$$
\theta_{\alpha \beta}^{\gamma}=\arccos \left(\hat{\boldsymbol{l}}_{\alpha \gamma} \cdot \hat{\boldsymbol{l}}_{\beta \gamma}\right) \text {. }
$$

The corresponding undeformed angle is denoted by $\Theta_{\alpha \beta}^{\gamma}=\arccos \left(\hat{\boldsymbol{L}}_{\alpha \gamma} \cdot \hat{\boldsymbol{L}}_{\beta \gamma}\right)$. Each angle is thus defined by a triple $(\alpha \beta)_{\gamma}$ for all nodes $\gamma \in \mathcal{N}$ with the set of all nodes $\mathcal{N}$.

We assume the potential energy of the truss decomposes additively into strain energy contributions from bar stretching and nodal deformations ${ }^{3}$. Therefore, bending deformation is accounted for by the angle changes at the nodes, which is most suitable for stretching-dominated truss lattices having high connectivities at nodes (Gibson and Ashby, 1999; Deshpande et al., 2001a,b). In order to account for finite rotations, we work in a corotational finitedeformation framework. The elastic energy of a bar $v \in \mathcal{B}$ of initial length $L_{v}$, stretched by an axial strain $\varepsilon_{v}$, and having energy density $W_{b}$ is

$$
W_{v}=W_{b}\left(\varepsilon_{v}, \boldsymbol{q}_{v}\right) V_{\mathrm{bar}, v} \quad \text { with } \quad \varepsilon_{v}=\left(l_{v}-L_{v}\right) / L_{v},
$$

where $\boldsymbol{q}_{v} \in \mathbb{R}^{p}$ represents a set of $p$ internal variables (required, e.g., in case of inelastic deformation mechanisms such as plasticity). $V_{\mathrm{bar}, v}=D_{v} L_{v}$ denotes the undeformed volume per thickness of each bar with thickness $D_{v}$ in two dimensions. For computational ease, the energy of a node $\gamma$ is approximated as the sum over all neighboring pairs $(\alpha \beta)_{\gamma}$ of bars meeting at that junction (Wang and Cuitiño, 2000; Pal et al., 2016), abbreviated by $(\alpha \beta) \in \mathcal{A}_{\gamma}$, i.e.,

$$
W_{\gamma}=\sum_{(\alpha \beta) \in \mathcal{A}_{\gamma}} W_{n}\left(\theta_{\alpha \beta}^{\gamma}-\Theta_{\alpha \beta}^{\gamma}\right) V_{\text {node }, \alpha \beta}^{\gamma},
$$

\footnotetext{
${ }^{2}$ We note that we adhere to standard trusses language here, while the approach is sufficiently general to apply to any discrete periodic networks. For example, "bars" more generally represent distance-dependent pair interactions.

${ }^{3}$ Beyond trusses, such a description is also common in atomistic systems with angle-dependent interaction potentials.
} 
where $W_{n}$ is the nodal energy density and $V_{\text {node }, \alpha \beta}^{\gamma}=D_{\alpha} D_{\beta}$ the effective volume per thickness of the undeformed node. These are assumptions on which our subsequent examples will be based. We point out that more accurate nodal and bar descriptions can be obtained, e.g., from full-detail FE simulations of individual nodes or bars (see, e.g., Meza et al. (2015), which showed that a linear elastic descriptions of nodes is feasible in the elastic regime) or from experiments (which is ongoing work). The above variational formulation is sufficiently general to apply to any discrete network description based on translational degrees of freedom; e.g., one can readily adjust (3) to account for more complex "multi-body" nodal energies or define alternative nodal volumes. Here, we focus on the extraction of a continuum model and thus demonstrate that - if a periodic network can be described by bar and nodal constitutive models such as those given above - then our micro-to-macro transition scheme yields an accurate continuum model and efficient computational implementation. Of course, ideally we would like to demonstrate its effectiveness for arbitrary underlying discrete lattices; however, the numerical examples require specific choices for the discrete lattice description.

The total potential energy of the truss is now the sum over all bar and nodal energies:

$$
I\left(\boldsymbol{x}_{\alpha}, \ldots, \boldsymbol{x}_{N_{n}}\right)=\sum_{v \in \mathcal{B}} W_{b}\left(\varepsilon_{\nu}, \boldsymbol{q}_{v}\right) V_{\mathrm{bar}, \nu}+\sum_{\gamma \in \mathcal{N}} \sum_{(\alpha \beta) \in \mathcal{A}_{\gamma}} W_{n}\left(\theta_{\alpha \beta}^{\gamma}-\Theta_{\alpha \beta}^{\gamma}\right) V_{\text {node }, \alpha \beta}^{\gamma} .
$$

Under quasistatic conditions, the equilibrium configuration for given boundary conditions is obtained by minimizing the total potential energy with respect to the nodal positions $\left\{\boldsymbol{x}_{\alpha}, \ldots, \boldsymbol{x}_{N_{n}}\right\}$. We deliberately set up a simple model system that allows us to study the response while controlling the relative contributions of stretching and bending deformations; this model can easily be extended and generalized into various directions, including three dimensions and more complex energy representations. Specific forms of $W_{b}$ and $W_{n}$ will be discussed in Section 2.3.

For our simulations, we will consider the two example lattice geometries shown in Fig. 1 (the methods presented here are sufficiently general for arbitrary truss architectures). First, the X-braced square lattice is obtained by periodically repeating the shown 5-node quadratic unit cell. For a square-shaped truss with $N$ unit cells per side length $H$, this results in a total of $N_{n}=12 N^{2}$ nodes and $N_{b}=6 N^{2}+2 N$ bars $\left(2 N^{2}+2 N\right.$ are horizontal/vertical and of length $L=H / N$, and $4 N^{2}$ are diagonal with length $L / \sqrt{2}$ ). Second, the shown triangular lattice is composed of equilateral triangles whose unit cell contains three nodes, resulting in a total of $N_{n}=6 N^{2}+3 N$ nodes and $N_{b}=3 N^{2}+\frac{7}{2} N$ bars (all of length $L$ ).

The relative density of a lattice is defined as the total volume of all bars and nodes normalized by the total volume occupied by the truss. Without loss of generality (and because it is a consequence of most deposition based fabrication techniques), we may assume that all bars have the same thickness $D_{v}=D$ for all $v \in \mathcal{B}$, and we denote by $d=D / L$ their slenderness ratio. In this case, the relative densities of the two lattices are given by

$$
\bar{\rho}_{\text {square }}=\left(\frac{4}{\sqrt{2}}+2\right) d, \quad \bar{\rho}_{\text {triangle }}=2 \sqrt{3} d .
$$

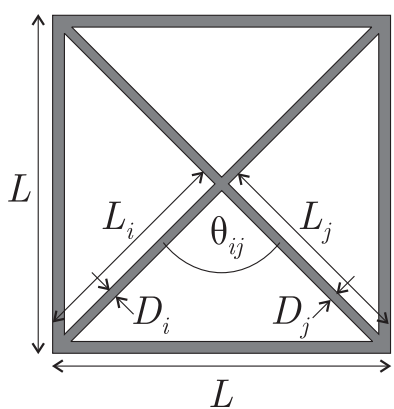

(a) square-type lattice unit cell

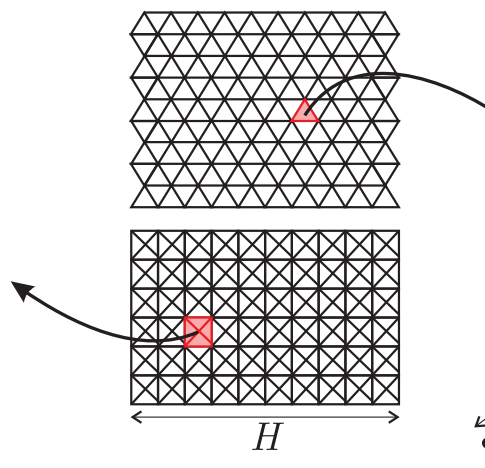

(b) truss lattices (c) triangular lattice unit cell

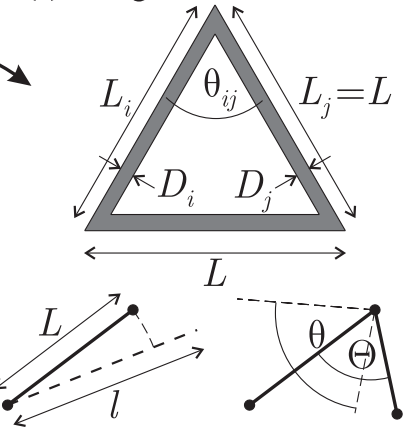

(d) angle and length changes

Figure 1: Schematic view of (a) square-type and (c) triangular truss unit cells, along with geometric definitions, which result in periodic truss lattices shown in (b); illustrations of bar stretching and angle changes with associated definitions are shown in (d). 


\subsection{Continuum model for truss lattices}

In order to model large trusses involving thousands to millions of bars and nodes, we introduce a continuum model for periodic truss networks which is similar in spirit to the homogenization approaches of Bardenhagen and Triantafyllidis (1994); Wang and Cuitiño (2000) and, in case of elastic trusses, results in a gradient elasticity formulation (Toupin, 1962; Koiter, 1964). To this end, we assume a separation of scales between the macroscopic truss and the underlying discrete lattice structure. On the macroscale, the continuum description defines a deformation mapping $\boldsymbol{x}=\boldsymbol{x}(\boldsymbol{X})$, where $\boldsymbol{x}$ and $\boldsymbol{X}$ are, respectively, the deformed and undeformed positions of a material point. By assuming continuous fields, the deformation within an infinitesimal neighborhood of a point $\boldsymbol{X}$ on the macroscale can be expanded into

$$
\boldsymbol{x}(\boldsymbol{X}+\mathrm{d} \boldsymbol{X})=\boldsymbol{x}(\boldsymbol{X})+\boldsymbol{F}(\boldsymbol{X}) \mathrm{d} \boldsymbol{X}+\frac{1}{2} \operatorname{Grad} \boldsymbol{F}(\mathrm{d} \boldsymbol{X} \otimes \mathrm{d} \boldsymbol{X})+\text { h.o.t. },
$$

where $\boldsymbol{F}(\boldsymbol{X})=\operatorname{Grad} \boldsymbol{x}$ denotes the deformation gradient tensor evaluated at $\boldsymbol{X}$.

In order to link the continuum description to the kinematics of the underlying discrete truss, we identify a representative unit cell (RUC) as shown in Fig. 2(d) and assume that the cell is significantly smaller than the macroscopic extensions of the truss (i.e., $L \ll H_{\alpha}$ ). A proper choice of the RUC must ensure that (i) all unique bars and angles are accounted for, (ii) without duplication, and (iii) the complete lattice results from periodic translation. As shown in Fig. 3, the square RUC is composed of $n_{b}=6$ bars and $n_{n}=12$ 2-bar junctions; the same applies to the triangular lattice whose unit cell includes the area of four elementary triangles. The volume per thickness of the square and triangular unit cells is $V_{\text {squ. }}=L^{2}$ and $V_{\text {tri. }}=\sqrt{3} L^{2}$, respectively, with unit cell side length $L$; in general, $V_{\mathrm{RUC}}=w L^{2}$ with a lattice-specific constant $w$.

Based on the separation of scales, we apply the Taylor expansion (6) to the deformation of the RUC, so that any point initially located at $\boldsymbol{X}$ (with respect to the RUC's center of mass) is deformed according to

$$
\boldsymbol{x}=\boldsymbol{x}_{0}+\boldsymbol{F} \boldsymbol{X}+\frac{1}{2} \operatorname{Grad} \boldsymbol{F}(\boldsymbol{X} \otimes \boldsymbol{X})+\text { h.o.t., }
$$

where $\boldsymbol{x}_{0}$ denotes the deformed position of the RUC's center of mass. Here and in the following, we will neglect all higher-order terms (h.o.t.). Note that for the separation of scales to hold, the deformation gradient may thus vary at most linearly at the RUC-level (which imposes length-scale constraints on phenomena to be described by the continuum model).

Let us denote by $\mathcal{B}_{\mathrm{RUC}}$ the set of all bars in the RUC, so that the deformed length of a bar $(\alpha, \beta) \in \mathcal{B}_{\mathrm{RUC}}$ which connects the nodes initially at $\boldsymbol{X}_{\alpha}$ and $\boldsymbol{X}_{\beta}$ according to the nonlocal expansion (7) becomes

$$
l_{\alpha \beta}=\left|\boldsymbol{x}_{\alpha}-\boldsymbol{x}_{\beta}\right|=\left|\boldsymbol{F} \boldsymbol{L}_{\alpha \beta}+\frac{1}{2} \operatorname{Grad} \boldsymbol{F}\left(\boldsymbol{X}_{\alpha} \otimes \boldsymbol{X}_{\alpha}-\boldsymbol{X}_{\beta} \otimes \boldsymbol{X}_{\beta}\right)\right| .
$$

Note that if we also neglect the quadratic term in the series expansion (or if the macroscopic deformation is uniform), we arrive at a local model which applies an affine deformation to the RUC, known as the Cauchy-Born rule (Ericksen,

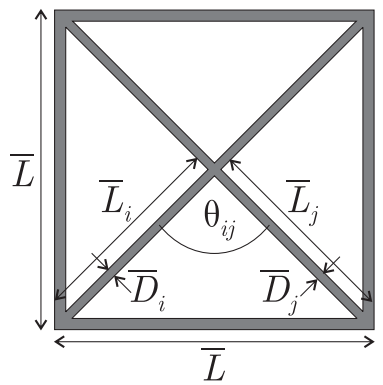

(a) discrete truss architecture

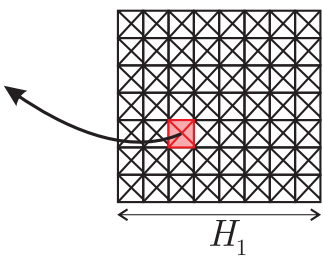

(b) discrete model

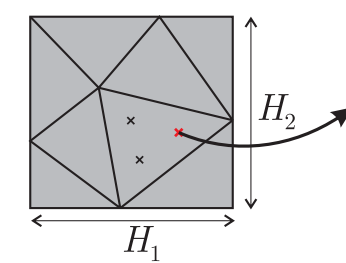

(c) continuum model

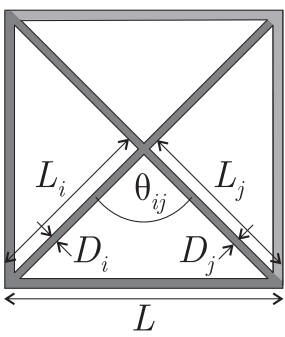

(d) representative unit cell

Figure 2: Discrete and continuum descriptions: (a) discrete truss architecture whose periodic repetition generates the truss lattice shown in (b). The continuum model (c) is based on the identification of a representative unit cell (RUC) shown in (d) for the square lattice. 
2008), i.e., $l_{\alpha \beta}=\left|\boldsymbol{F} \boldsymbol{L}_{\alpha \beta}\right|$. The nonlocal expansion (8) is also known as the extended (or higher-order) Cauchy-Born rule in the study of atomistic lattices; see, e.g., Sunyk and Steinmann (2003); Sun and Liew (2008).

Introducing directional unit vectors such that $\boldsymbol{L}_{\alpha \beta}=L_{\alpha \beta} \hat{\boldsymbol{L}}_{\alpha \beta}$ and using $\boldsymbol{X}_{\beta}=\boldsymbol{X}_{\alpha}-\boldsymbol{L}_{\alpha \beta}$ allows us to rewrite (8) as

$$
l_{\alpha \beta}=\left|L_{\alpha \beta} \boldsymbol{F} \hat{\boldsymbol{L}}_{\alpha \beta}+\frac{1}{2} \operatorname{Grad} \boldsymbol{F}\left[L_{\alpha \beta}\left(\hat{\boldsymbol{L}}_{\alpha \beta} \otimes \boldsymbol{X}_{\alpha}+\boldsymbol{X}_{\alpha} \otimes \hat{\boldsymbol{L}}_{\alpha \beta}\right)-L_{\alpha \beta}^{2} \hat{\boldsymbol{L}}_{\alpha \beta} \otimes \hat{\boldsymbol{L}}_{\alpha \beta}\right]\right|
$$

Since the coordinate origin is arbitrary and irrelevant to the deformed lengths, the axial engineering strain of a bar $v \in \mathcal{B}_{\mathrm{RUC}}$, defined in (2), now follows as

$$
\varepsilon_{v}=\frac{l_{v}}{L_{v}}-1=\left|\boldsymbol{F} \hat{\boldsymbol{L}}_{v}-\frac{L_{v}}{2} \operatorname{Grad} \boldsymbol{F}\left(\hat{\boldsymbol{L}}_{v} \otimes \hat{\boldsymbol{L}}_{v}\right)\right|-1 .
$$

Note that, in contrast to the local model, the strain (10) depends on the choice of the RUC size, which can be used as a control parameter for the nonlocal effects (within the limits imposed by the separation-of-scales assumption). In case of affine deformation ( $\operatorname{Grad} \boldsymbol{F}=\mathbf{0})$ or in the limit of a vanishing RUC size $\left(L_{v} \rightarrow 0\right)$, we recover the Cauchy-Born rule with engineering strain $\varepsilon_{v}=\left(\hat{\boldsymbol{L}}_{v} \cdot \boldsymbol{C} \hat{\boldsymbol{L}}_{v}\right)^{1 / 2}-1$, where $\boldsymbol{C}=\boldsymbol{F}^{\mathrm{T}} \boldsymbol{F}$ denotes the right Cauchy-Green tensor.

Similarly, using (1), the deformed angle between the two bars $(\alpha, \gamma),(\beta, \gamma) \in \mathcal{B}_{\mathrm{RUC}}$ for a node $\gamma \in \mathcal{N}_{\mathrm{RUC}}$ (where $\mathcal{N}_{\text {RUC }}$ denotes the set of all nodes in the RUC) is obtained from the deformed unit vectors, viz.

$$
\theta_{\alpha \beta}^{\gamma}=\arccos \left(\hat{\boldsymbol{l}}_{\alpha \gamma} \cdot \hat{\boldsymbol{l}}_{\beta \gamma}\right) \quad \text { with } \quad \hat{\boldsymbol{l}}_{v}=\frac{L_{v}}{l_{v}}\left[\boldsymbol{F} \hat{\boldsymbol{L}}_{v}-\frac{L_{v}}{2} \operatorname{Grad} \boldsymbol{F}\left(\hat{\boldsymbol{L}}_{v} \otimes \hat{\boldsymbol{L}}_{v}\right)\right] .
$$

The associated undeformed angle is $\Theta_{\alpha \beta}^{\gamma}=\arccos \left(\hat{\boldsymbol{L}}_{\alpha \gamma} \cdot \hat{\boldsymbol{L}}_{\beta \gamma}\right)$. Obviously, the angular changes also depend on the unit cell size and possess a finite value in the affine limit of a vanishing unit cell size.

Based on the above unit cell deformation, the continuum energy density is defined by

$$
W(\boldsymbol{F}, \operatorname{Grad} \boldsymbol{F})=\frac{1}{V_{\mathrm{RUC}}}\left[\sum_{v \in \mathcal{B}_{\mathrm{RUC}}} W_{b}\left(\varepsilon_{v}, \boldsymbol{q}_{v}\right) L_{v} D_{v}+\sum_{\gamma \in \mathcal{N}_{\mathrm{RUC}}} \sum_{(\alpha \beta) \in \mathcal{A} \gamma} W_{n}\left(\theta_{\alpha \beta}^{\gamma}-\Theta_{\alpha \beta}^{\gamma}\right) D_{\alpha} D_{\beta}\right],
$$

where $\varepsilon_{v}$ and $\theta_{\alpha \beta}^{\gamma}$ are obtained from applying (10) and (11) to all bars and nodal junctions in the RUC. Since the individual bar lengths and thicknesses are proportional to the unit cell extension $\left(L_{v} \propto L, D_{v}=d_{v} L \propto L\right)$ and $V_{\mathrm{RUC}} \propto L^{2}$, the above energy density is independent of the choice of $L$ under affine deformations, as required. In addition, consider a discrete truss as described in Section 2.1, having volume per thickness $V_{\text {truss }}=H_{1} H_{2}$ (see Fig. 2) and being composed of $N_{b}$ bars $v \in \mathcal{B}$ and $N_{n}$ nodes $\gamma \in \mathcal{N}$. Its total strain energy is given by

$$
I=\sum_{v \in \mathcal{B}} W_{b}\left(\varepsilon_{v}\right) \bar{L}_{v} \bar{D}_{v}+\sum_{\gamma \in \mathcal{N}} \sum_{(\alpha \beta) \in \mathcal{A} \gamma} W_{n}\left(\theta_{\alpha \beta}^{\gamma}-\Theta_{\alpha \beta}^{\gamma}\right) \bar{D}_{\alpha} \bar{D}_{\beta}
$$
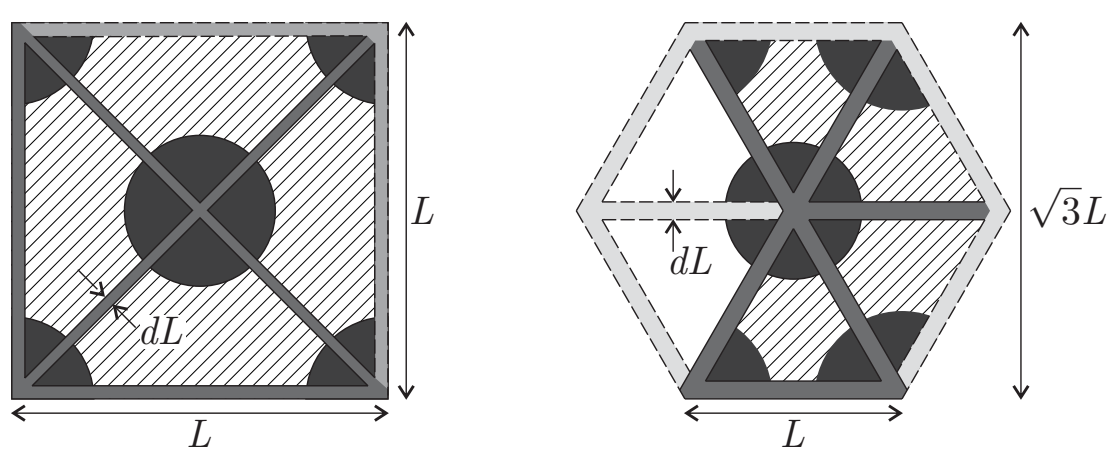

Figure 3: Representative unit cells chosen for the square and triangular lattices: $n_{b}=6$ unique bars and $n_{n}=12$ unique 2-bar junctions are used to compute the energy of the respective RUC are shown in dark colors; RUC volumes (per thickness) are shown as hatched areas. 
Note that, here and in the following, we denote the beam dimensions of the actual, discrete truss by $\bar{L}_{v}$ and $\bar{D}_{v}$ to differentiate the physical truss geometry from that of the fictitious RUC, see Fig. 2. It is a simple exercise to show that under affine deformations the exact energy (13) indeed coincides with $V_{\text {truss }} W(\boldsymbol{F}, \mathbf{0})$ from (12) for the chosen RUCs, because $V_{\text {truss }} / V_{\mathrm{RUC}}$ is the number of unit cells inside the truss, and each unit cell contributes the same energy under affine deformations (this also confirms our choices of the RUCs). If the deformation is no longer affine, then the size of the RUC (i.e., the choice of $L$ ) affects the solution.

The components of the first Piola-Kirchhoff stress tensor and the third-order couple stress tensor are obtained from the nonlocal energy density (12) as, respectively,

$$
P_{i J}=\frac{\partial W}{\partial F_{i J}}(\boldsymbol{F}, \operatorname{Grad} \boldsymbol{F}), \quad Y_{i J K}=\frac{\partial W}{\partial F_{i J, K}}(\boldsymbol{F}, \operatorname{Grad} \boldsymbol{F}) .
$$

The first Piola-Kirchhoff stress tensor (with beam slenderness ratios $d_{v}=D_{v} / L$ ) is evaluated as

$$
\boldsymbol{P}=\frac{1}{w}\left[\sum_{\nu \in \mathcal{B}_{\mathrm{RUC}}} W_{b}^{\prime}\left(\varepsilon_{v}, \boldsymbol{q}_{v}\right) d_{\nu} \frac{L_{v}}{L} \frac{\partial \varepsilon_{v}}{\partial \boldsymbol{F}}+\sum_{\gamma \in \mathcal{N}_{\mathrm{RUC}}} \sum_{(\alpha \beta) \in \mathcal{A}_{\gamma}} W_{n}^{\prime}\left(\theta_{\alpha \beta}^{\gamma}-\Theta_{\alpha \beta}^{\gamma}\right) d_{\alpha} d_{\beta} \frac{\partial \theta_{\alpha \beta}^{\gamma}}{\partial \boldsymbol{F}}\right] .
$$

with partial derivatives

$$
\frac{\partial \varepsilon_{v}}{\partial \boldsymbol{F}}=\hat{\boldsymbol{l}}_{v} \otimes \hat{\boldsymbol{L}}_{v}, \quad \frac{\partial \theta_{\alpha \beta}^{\gamma}}{\partial \boldsymbol{F}}=-\frac{1}{\sin \theta_{\alpha \beta}^{\gamma}}\left[\frac{1}{l_{\gamma \alpha}}\left(\hat{\boldsymbol{l}}_{\gamma \beta}-\hat{\boldsymbol{l}}_{\gamma \alpha} \cos \theta_{\alpha \beta}^{\gamma}\right) \otimes \boldsymbol{L}_{\gamma \alpha}+\frac{1}{l_{\gamma \beta}}\left(\hat{\boldsymbol{l}}_{\gamma \alpha}-\hat{\boldsymbol{l}}_{\gamma \beta} \cos \theta_{\alpha \beta}^{\gamma}\right) \otimes \boldsymbol{L}_{\gamma \beta}\right] .
$$

Next, writing $l_{i}^{v}=\left(\boldsymbol{l}_{v}\right)_{i}$ for the $i$ th component of vector $\boldsymbol{l}_{v}$ to avoid confusion, the components of the incremental stiffness tensor in index notation are obtained as

$$
\begin{aligned}
\mathbb{C}_{i J k L}=\frac{\partial^{2} W}{\partial F_{i J} \partial F_{k L}}=\frac{1}{w}\{ & \sum_{v \in \mathcal{B}_{\mathrm{RUC}}} d_{v} \frac{L_{v}}{L}\left[W_{b}^{\prime}\left(\varepsilon_{v}, \boldsymbol{q}_{v}\right) \frac{L_{v}}{l_{v}}\left(\delta_{i k}-\hat{l}_{i}^{v} \hat{l}_{k}^{v}\right)+W_{b}^{\prime \prime}\left(\varepsilon_{v}, \boldsymbol{q}_{v}\right) \hat{l}_{i}^{\gamma} \hat{l}_{k}^{v}\right] \hat{L}_{J}^{v} \hat{L}_{L}^{v} \\
& \left.+\sum_{\gamma \in \mathcal{N}_{\mathrm{RUC}}} \sum_{(\alpha \beta) \in \mathcal{F}_{\gamma}} d_{\alpha} d_{\beta}\left[W_{n}^{\prime}\left(\theta_{\alpha \beta}^{\gamma}-\Theta_{\alpha \beta}^{\gamma}\right) \frac{\partial^{2} \theta_{\alpha \beta}^{\gamma}}{\partial F_{i J} \partial F_{k L}}+W_{n}^{\prime \prime}\left(\theta_{\alpha \beta}^{\gamma}-\Theta_{\alpha \beta}^{\gamma}\right) \frac{\partial \theta_{\alpha \beta}^{\gamma}}{\partial F_{i J}} \frac{\partial \theta_{\alpha \beta}^{\gamma}}{\partial F_{k L}}\right]\right\}
\end{aligned}
$$

with all partial derivatives obtained in an analogous fashion (not written out here for conciseness). Similarly, $\boldsymbol{Y}$ and the higher-order stiffness tensors associated with $\boldsymbol{Y}$ can be obtained by differentiation, using

$$
\frac{\partial \varepsilon_{v}}{\partial \operatorname{Grad} \boldsymbol{F}}=-\frac{L_{v}}{2} \hat{\boldsymbol{l}}_{v} \otimes \hat{\boldsymbol{L}}_{v} \otimes \hat{\boldsymbol{L}}_{v}
$$

and

$$
\frac{\partial \theta_{\alpha \beta}^{\gamma}}{\partial \operatorname{Grad} \boldsymbol{F}}=\frac{1}{\sin \theta_{\alpha \beta}^{\gamma}}\left[\frac{1}{l_{\alpha \gamma}}\left(\frac{1}{2} \hat{\boldsymbol{l}}_{\beta \gamma}-\hat{\boldsymbol{l}}_{\alpha \gamma} \cos \theta_{\alpha \beta}^{\gamma}\right) \otimes \boldsymbol{L}_{\alpha \gamma} \otimes \boldsymbol{L}_{\alpha \gamma}+\frac{1}{l_{\beta \gamma}}\left(\frac{1}{2} \hat{\boldsymbol{l}}_{\alpha \gamma}-\hat{\boldsymbol{l}}_{\beta \gamma} \cos \theta_{\alpha \beta}^{\gamma}\right) \otimes \boldsymbol{L}_{\beta \gamma} \otimes \boldsymbol{L}_{\beta \gamma}\right] .
$$

We note that in case of inelastic material behavior, the above derivatives of the energy densities denote the total derivatives, e.g.,

$$
W_{b}^{\prime}(\varepsilon, \boldsymbol{q})=\frac{\mathrm{d} W_{b}}{\mathrm{~d} \varepsilon}(\varepsilon, \boldsymbol{q})=\frac{\partial W_{b}}{\partial \varepsilon}(\varepsilon, \boldsymbol{q})+\frac{\partial W_{b}}{\partial \boldsymbol{q}}(\varepsilon, \boldsymbol{q}) \cdot \frac{\partial \boldsymbol{q}}{\partial \varepsilon}
$$

The macroscopic boundary value problem can be described by a variational principle, starting with the total potential energy functional of a body $\Omega$, expressed in the Lagrangian configuration, i.e.,

$$
I[\boldsymbol{u}]=\int_{\Omega} W(\boldsymbol{F}, \operatorname{Grad} \boldsymbol{F}) \mathrm{d} V-\int_{\Omega} R \boldsymbol{B} \cdot \boldsymbol{u} \mathrm{d} V-\int_{\partial \Omega_{T}} \boldsymbol{T} \cdot \boldsymbol{u} \mathrm{d} S-\int_{\partial \Omega_{M}} \boldsymbol{M} \cdot(\operatorname{Grad} \boldsymbol{u}) \boldsymbol{N} \mathrm{d} S,
$$

with Lagrangian mass density $R$, body forces $\boldsymbol{B}$, and the displacement field $\boldsymbol{u}(\boldsymbol{X})=\boldsymbol{x}(\boldsymbol{X})-\boldsymbol{X}$. Essential boundary conditions $\boldsymbol{u}=\boldsymbol{u}_{0}$ are enforced on $\partial \Omega_{u}$, surface tractions $\boldsymbol{T}$ on $\partial \Omega_{T}$, and surface moments $\boldsymbol{M}$ on $\partial \Omega_{M}$ (for physical reasons, we assume that no surface gradients are imposed as essential boundary conditions). Further, for simplicity we consider only smooth surfaces and neglect edge or corner effects; see, e.g., Rudraraju et al. (2014) for a discussion. By computing the first variation of (21), applying the divergence theorem and enforcing that $\boldsymbol{u}=\arg \min \left\{I[\boldsymbol{u}] \mid \boldsymbol{u}=\boldsymbol{u}_{0}\right.$ on $\left.\partial \Omega_{u}\right\}$, one arrives at the linear momentum balance equation $P_{i J J}^{*}+R B_{i}=0$ in $\Omega$ with the effective stress tensor components $P_{i J}^{*}=P_{i J}-Y_{i J K, K}$ and associated natural boundary conditions, see Toupin (1962, 1964); Koiter (1964) for details. 


\subsection{Specific forms of stretching and nodal constitutive laws}

The above formulation is sufficiently general to work with any choice of the strain energy densities for bars and nodes, $W_{b}(\varepsilon)$ and $W_{n}(\Delta \theta)$, respectively. For our numerical examples, we will use the following specific forms.

First, for a purely elastic response, we consider the strain energy density of uniaxially-stretched bars along with a quadratic elastic energy density for the nodes (Kirkwood, 1939):

$$
W_{b}(\varepsilon)=\frac{E}{2} \varepsilon^{2}, \quad W_{n}(\Delta \theta)=\frac{K}{2}(\Delta \theta)^{2}
$$

$E$ and $K$ are the bar's Young modulus and the nodal bending stiffness, respectively. The ratio $E / K$ can be exploited to control the relative importance of the two deformation mechanisms. For example, for $E / K \rightarrow 0$ the nodes become rigid and suppress angular changes. By contrast, if $E / K \rightarrow \infty$, the bars become rigid and all deformation is carried by angular changes. Unfortunately, little is known about the inelastic deformation of truss junctions (also, we do not intend to model a particular material system here). Therefore, in the following we will make use of the above form of $W_{n}$ and build inelastic mechanisms into $W_{b}$. We note that the energies (22) can also be interpreted as describing linear elastic, corotational beams that combine stretching and bending deformation (Desmoulins et al., 2016).

As an alternative to the above elastic material models, we use an elastic-plastic description for the bars. Following the concept of variational constitutive updates (Ortiz and Stainier, 1999), we introduce internal (history) variables $\varepsilon^{p}$ (the plastic strain) and $e$ (the accumulated plastic strain) for each bar, i.e., $\boldsymbol{q}=\left(\varepsilon^{p}, e\right)$. We use constant time increments $\Delta t=t_{n+1}-t_{n}>0$ and we write $\boldsymbol{q}_{n}=\boldsymbol{q}\left(t_{n}\right)$ for the internal variables at time step $n$. Assume an elasticplastic incremental potential

$$
\Psi\left(\varepsilon_{n+1}, \varepsilon_{n+1}^{p}, \varepsilon_{n}^{p}, e_{n}\right)=\frac{E}{2}\left(\varepsilon_{n+1}-\varepsilon_{n+1}^{p}\right)^{2}+S e_{n+1}^{2}+\Delta t \tau_{0}\left|\frac{\varepsilon_{n+1}^{p}-\varepsilon_{n}^{p}}{\Delta t}\right|,
$$

where the first term represents the elastic energy at $t_{n+1}$ (with Young's modulus $E$ ), the second term represents linear plastic hardening with hardening modulus $S$, and the final term defines the incremental dissipation distance (Mielke, 2002; Carstensen et al., 2002) for rate-independent plasticity with $\tau_{0}$ being the initial yield stress. The effective incremental energy density of a bar is then defined as (Kochmann and Amelang, 2016)

$$
W_{b}\left(\varepsilon_{n+1}, \boldsymbol{q}_{n}\right)=\inf _{\varepsilon_{n+1}^{p}} \Psi\left(\varepsilon_{n+1}, \varepsilon_{n+1}^{p}, \varepsilon_{n}^{p}, e_{n}\right) .
$$

Minimization in (24) can be carried out analytically by solving the stationarity equation. This yields the incremental kinetic law for the plastic strain:

$$
\varepsilon_{n+1}^{p}\left(\varepsilon_{n+1}, \varepsilon_{n}^{p}, e_{n}\right)=\varepsilon_{n}^{p}+\frac{E\left|\varepsilon_{n+1}-\varepsilon_{n}^{p}\right|-\left(\tau_{0}+2 S e_{n}\right)}{E+2 S} \operatorname{sign}\left(\varepsilon_{n+1}-\varepsilon_{n}^{p}\right)
$$

if $E\left|\varepsilon_{n+1}-\varepsilon_{n}^{p}\right|-\left(\tau_{0}+2 S e_{n}\right)>0$ (plastic step), and $\varepsilon_{n+1}^{p}=\varepsilon_{n}^{p}$ otherwise (elastic step). The new internal variables are thus $\boldsymbol{q}_{n+1}=\left(\varepsilon_{n+1}^{p}, e_{n+1}\right)$ with $e_{n+1}=e_{n}+\left|\varepsilon_{n+1}^{p}-\varepsilon_{n}^{p}\right|$.

For example, for monotonic tensile loading this model results in a piecewise-linear stress-strain response:

$$
\sigma=W_{b}^{\prime}(\varepsilon)= \begin{cases}E \varepsilon, & \text { if } \varepsilon \leq \tau_{0} / E, \\ \tau_{0}+2 S /(E+2 S)\left(E \varepsilon-\tau_{0}\right), & \text { if } \varepsilon>\tau_{0} / E,\end{cases}
$$

and general loading can be derived analogously. To account for bar failure, this model is extended by introducing a failure criterion. To this end, we define a critical strain $\varepsilon_{\mathrm{cr}}$ and irreversibly eliminate those bars whose axial strain reaches $\varepsilon_{\mathrm{cr}}$.

\section{Numerical implementation}

\subsection{Finite element formulation of the nonlocal continuum model}

The above nonlocal continuum model is implemented in a finite element (FE) framework. The variational structure of the problem admits a convenient numerical treatment. Starting with the potential energy functional (21), we compute the first variation with respect to all admissible perturbations $\boldsymbol{\eta} \in \mathcal{E}$ in the function space $\mathcal{E}=\left\{\boldsymbol{\eta} \mid \boldsymbol{\eta}=\mathbf{0}\right.$ on $\left.\partial \Omega_{u}\right\}$ 
to find the solution $\boldsymbol{u} \in \mathcal{U}$ with $\mathcal{U}=\left\{\boldsymbol{u} \mid \boldsymbol{u}=\boldsymbol{u}_{0}\right.$ on $\left.\partial \Omega_{u}\right\}$. The first variation of (21) thus yields

$$
\begin{aligned}
\delta I[\boldsymbol{u}, \boldsymbol{\eta}]=\int_{\Omega} & {\left[\frac{\partial W}{\partial F_{i J}}(\boldsymbol{F}, \operatorname{Grad} \boldsymbol{F}) \eta_{i, J}+\frac{\partial W}{\partial F_{i J, K}}(\boldsymbol{F}, \operatorname{Grad} \boldsymbol{F}) \eta_{i, J K}\right] \mathrm{d} V } \\
& -\int_{\Omega} R B_{i} \eta_{i} \mathrm{~d} V-\int_{\partial \Omega_{T}} T_{i} \eta_{i} \mathrm{~d} S-\int_{\partial \Omega_{M}} M_{i} \eta_{i, J} N_{J} \mathrm{~d} S=0,
\end{aligned}
$$

where we use standard indicial notation with the Einstein summation convention. The Galerkin weak form is obtained by introducing approximate (finite-dimensional) functional spaces

$$
\mathcal{U}^{h}=\left\{\boldsymbol{u}^{h} \in \mathcal{H}^{2}(\Omega) \mid \boldsymbol{u}^{h}=\boldsymbol{u}_{0} \text { on } \partial \Omega_{u}\right\} \quad \text { and } \quad \mathcal{E}^{h}=\left\{\boldsymbol{\eta}^{h} \in \mathcal{H}^{2}(\Omega) \mid \boldsymbol{\eta}^{h}=\mathbf{0} \text { on } \partial \Omega_{u}\right\} .
$$

The second derivatives in (27) require that $\boldsymbol{u}^{h}, \boldsymbol{\eta}^{h} \in \mathcal{H}^{2}(\Omega)$. We thus arrive at

$$
\int_{\Omega}\left[\frac{\partial W}{\partial F_{i J}}\left(\boldsymbol{F}^{h}, \operatorname{Grad} \boldsymbol{F}^{h}\right) \eta_{i, J}^{h}+\frac{\partial W}{\partial F_{i J, K}}\left(\boldsymbol{F}^{h}, \operatorname{Grad} \boldsymbol{F}^{h}\right) \eta_{i, J K}^{h}\right] \mathrm{d} V-\int_{\Omega} R B_{i} \eta_{i}^{h} \mathrm{~d} V-\int_{\partial \Omega_{T}} T_{i} \eta_{i}^{h} \mathrm{~d} S-\int_{\partial \Omega_{M}} M_{i} \eta_{i, J}^{h} N_{J} \mathrm{~d} S=0 .
$$

We discretize the spatial fields by admissible shape functions $N^{a}\left(a=1, \ldots, n_{s}\right)$ and associated nodal displacements $\boldsymbol{U}=\left\{\boldsymbol{u}^{1}, \ldots, \boldsymbol{u}^{n_{s}}\right\}$ and perturbations $\boldsymbol{E}=\left\{\boldsymbol{\eta}^{1}, \ldots, \boldsymbol{\eta}^{n_{s}}\right\}$ such that

$$
\boldsymbol{u}^{h}(\boldsymbol{X})=\sum_{a=1}^{n_{s}} \boldsymbol{u}^{a} N^{a}(\boldsymbol{X}), \quad \boldsymbol{\eta}^{h}(\boldsymbol{X})=\sum_{a=1}^{n_{s}} \boldsymbol{\eta}^{a} N^{a}(\boldsymbol{X})
$$

With positions $\boldsymbol{x}^{a}=\boldsymbol{X}^{a}+\boldsymbol{u}^{a}$ in a Lagrangian frame of reference, we obtain the first and second deformation gradients

$$
F_{i J}^{h}=x_{i, J}=\delta_{i J}+\sum_{a} u_{i}^{a} N_{, J}^{a}(\boldsymbol{X}), \quad\left(\operatorname{Grad} \boldsymbol{F}^{h}\right)_{i J K}=F_{i J, K}^{h}=\sum_{a} u_{i}^{a} N_{, J K}^{a}(\boldsymbol{X}) .
$$

Insertion of expressions (31) into (29) along with the definitions (14) and using the fact that (29) must hold for all variations $\boldsymbol{\eta}^{h} \in \mathcal{E}$ results in the nonlinear (quasistatic) governing equation $\boldsymbol{F}_{\text {int }}(\boldsymbol{U})-\boldsymbol{F}_{\text {ext }}=\mathbf{0}$ with the global internal and external force vectors, respectively, defined by

$$
\begin{aligned}
& F_{\text {int }, i}^{a}=\int_{\Omega}\left[P_{i J}\left(\boldsymbol{F}^{h}, \operatorname{Grad} \boldsymbol{F}^{h}\right) N_{, J}^{a}+Y_{i J K}\left(\boldsymbol{F}^{h}, \operatorname{Grad} \boldsymbol{F}^{h}\right) N_{, J K}^{a}\right] \mathrm{d} V, \\
& F_{\mathrm{ext}, i}^{a}=\int_{\Omega} R B_{i} N^{a} \mathrm{~d} V+\int_{\partial \Omega_{T}} T_{i} N^{a} \mathrm{~d} S+\int_{\partial \Omega_{M}} M_{i} N_{, J}^{a} N_{J} \mathrm{~d} S,
\end{aligned}
$$

where we conveniently wrote $F_{i}^{a}$ for the $i$ th component of force vector $\boldsymbol{F}^{a}$, and the global vectors are defined as, e.g., $\boldsymbol{F}_{\text {int }}=\left\{\boldsymbol{F}_{\text {int }}^{1}, \ldots, \boldsymbol{F}_{\text {int }}^{n_{s}}\right\}$. The second derivatives of the shape functions appearing in (31) and (32) require that $N^{a}(\boldsymbol{X}) \in \mathcal{H}^{2}\left(\Omega^{h}\right)$. Here, we choose isoparametric triangular plane-strain elements with quadratic interpolation to represent the approximate body $\Omega^{h}$; see Appendix A for a derivation of the shape function derivatives.

The nonlinear system is solved by Newton-Raphson iteration which, in turn, requires the calculation of the incremental tangent matrix whose components are

$$
K_{\mathrm{int}, i j}^{a b}=\frac{\partial F_{\mathrm{int}, i}^{a}}{\partial u_{j}^{b}}=\int_{\Omega}\left[\frac{\partial P_{i J}}{\partial F_{j K}} N_{, J}^{a} N_{, K}^{b}+\frac{\partial P_{i J}}{\partial F_{j K, L}} N_{, J}^{a} N_{, K L}^{b}+\frac{\partial Y_{i J K}}{\partial F_{j L}} N_{, J K}^{a} N_{, L}^{b}+\frac{\partial Y_{i J K}}{\partial F_{j L, M}} N_{, J K}^{a} N_{, L M}^{b}\right] \mathrm{d} V .
$$

The nonlocal material model discussed in Section 2.2 provides the components of the first- and second-order stiffness tensors, i.e.,

$$
\mathbb{C}_{i J j K}=\frac{\partial P_{i J}}{\partial F_{j K}}, \quad K_{i J j K L}=\frac{\partial P_{i J}}{\partial F_{j K, L}}, \quad K_{i J K j L}=\frac{\partial Y_{i J K}}{\partial F_{j L}}, \quad K_{i J K j L M}=\frac{\partial Y_{i J K}}{\partial F_{j L, M}} .
$$

Note that by definition we have the major symmetries $\mathbb{C}_{i J j K}=\mathbb{C}_{j K i J}, K_{i J j K L}=K_{j K L i J}$ and $K_{i J K j L M}=K_{j L M i J K}$ as well as the minor symmetries $K_{i J j K L}=K_{i J j L K}$ and $K_{i J K j L M}=K_{i J K j L M}=K_{i J K j M L}=K_{i K J j M L}$. If the local model is used (i.e., if the RUC size $L \rightarrow 0$ in the nonlocal model), then all higher-gradient terms in the above derivation vanish and one recovers the classical nonlinear FE formulation with the affine Cauchy-Born rule applied to the RUC. 


\subsection{Nonlocal continuum formulation at the RUC-level}

From a computational perspective, it is convenient to notice that all continuum quantities required at the quadraturepoint level of an FE formulation (such as the energy density, stress and incremental tangent tensors) are derived from the deformation of a truss unit cell. Therefore, if an FE implementation of the discrete truss model is already available, it can be conveniently re-used to obtain the above quantities - like in the $\mathrm{FE}^{2}$ method (Feyel and Chaboche, 2000; Miehe et al., 2002) with strain-driven affine micro-deformation (in fact, the case of affine RUC deformation in the $\mathrm{FE}^{2}$ approach corresponds to our local model).

Consider an FE discretization of the RUC whose nodal displacements are denoted $\boldsymbol{U}_{\mathrm{RUC}}=\left\{\boldsymbol{u}_{1}, \ldots, \boldsymbol{u}_{n_{n}}\right\}$, assuming there are $n_{n}$ nodes and $n_{e}$ elements in the RUC. The total potential energy of a RUC is hence

$$
I_{\mathrm{RUC}}(\boldsymbol{V})=\sum_{a=1}^{n_{e}} W_{a}\left(\boldsymbol{U}_{\mathrm{RUC}}\right),
$$

where $W_{a}$ is the energy contributed by element $a$. The nonlocal Cauchy-Born rule (7) links the nodal position $\boldsymbol{x}_{i}=$ $\boldsymbol{X}_{i}+\boldsymbol{v}_{i}$ to the average deformation gradient $\boldsymbol{F}$ and second gradient $\operatorname{Grad} \boldsymbol{F}$. Therefore, the effective stress tensor of the RUC is obtained via

$$
P_{i J}=\frac{\partial I_{\mathrm{RUC}}}{\partial F_{i J}}=\sum_{a=1}^{n_{e}} \frac{\partial W_{a}}{\partial v_{k}^{j}}\left(\boldsymbol{U}_{\mathrm{RUC}}\right) \frac{\partial v_{k}^{j}}{\partial F_{i J}}=\sum_{j=1}^{n_{n}} f_{i}^{j}\left(\boldsymbol{U}_{\mathrm{RUC}}\right) X_{J}^{j},
$$

where $f_{i}^{j}$ denotes the $i$ th component of the total force vector acting on node $j$ (to avoid ambiguity, we again denote by $u_{i}^{j}$ the $i$ th component of $\boldsymbol{u}_{j}$ ). Analogously, the third-order stress tensor has components

$$
Y_{i J K}=\frac{\partial I_{\mathrm{RUC}}}{\partial F_{i J, K}}=\sum_{a=1}^{n_{e}} \frac{\partial W_{a}}{\partial v_{k}^{j}}\left(\boldsymbol{U}_{\mathrm{RUC}}\right) \frac{\partial v_{k}^{j}}{\partial F_{i J, K}}=\sum_{j=1}^{n_{n}} f_{i}^{j}\left(\boldsymbol{U}_{\mathrm{RUC}}\right) X_{J}^{j} X_{K}^{j} .
$$

The corresponding stiffness tensors (34) are obtained by differentiation, giving

$$
\mathbb{C}_{i J k L}=\frac{\partial P_{i J}}{\partial F_{k L}}=\frac{\partial}{\partial F_{k L}} \sum_{j=1}^{n_{n}} f_{i}^{j}\left(\boldsymbol{U}_{\mathrm{RUC}}\right) X_{J}^{j}=\sum_{j, l=1}^{n_{n}} k_{i k}^{j l}\left(\boldsymbol{U}_{\mathrm{RUC}}\right) X_{J}^{j} X_{L}^{l} \quad \text { where } \quad k_{i k}^{j l}=\frac{\partial f_{i}^{j}}{\partial u_{k}^{l}}
$$

is the component of the total RUC stiffness matrix associated with $u_{i}^{j}$ and $u_{k}^{l}$. Similarly, the fifth- and sixth-order stiffness tensors are calculated as

$$
K_{i J k M N}=\sum_{j, l=1}^{n_{n}} k_{i k}^{j l}\left(\boldsymbol{U}_{\mathrm{RUC}}\right) X_{J}^{j} X_{M}^{l} X_{N}^{l}, \quad K_{i J M k N}=\sum_{j, l=1}^{n_{n}} k_{i k}^{j l}\left(\boldsymbol{U}_{\mathrm{RUC}}\right) X_{J}^{j} X_{M}^{j} X_{N}^{l}, \quad K_{i J K I M N}=\sum_{j, o=1}^{n_{n}} k_{i l}^{j o}\left(\boldsymbol{U}_{\mathrm{RUC}}\right) X_{J}^{j} X_{K}^{j} X_{M}^{o} X_{N}^{o},
$$

which satisfy the symmetries discussed in Section 3.1. Recall that at the RUC-level there is no need to solve for $\boldsymbol{U}_{\mathrm{RUC}}$ since these displacements are known from the second-order homogenization scheme. However, the above expressions allow for a simple calculation of the quantities of interest for given $\boldsymbol{U}_{\mathrm{RUC}}$.

\section{Results}

\subsection{Material-point calculations with the local model}

Before presenting finite element solutions to boundary value problems, let us illustrate the constitutive response of the local continuum model by a material point calculation. For example, consider the X-braced square lattice undergoing uniform uniaxial compression with deformation gradient $\boldsymbol{F}=\boldsymbol{I}+\boldsymbol{\varepsilon}_{y y} \boldsymbol{e}_{y} \otimes \boldsymbol{e}_{y}$ parametrized by the only non-zero strain component $\varepsilon_{y y} \leq 0$. Here and in the following, we assume a uniform beam slenderness ratio $d=0.1$, 
(a) total stresses (elastic)

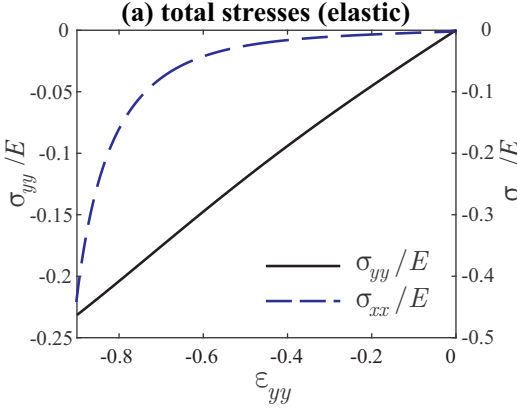

(d) total stresses (inelastic)

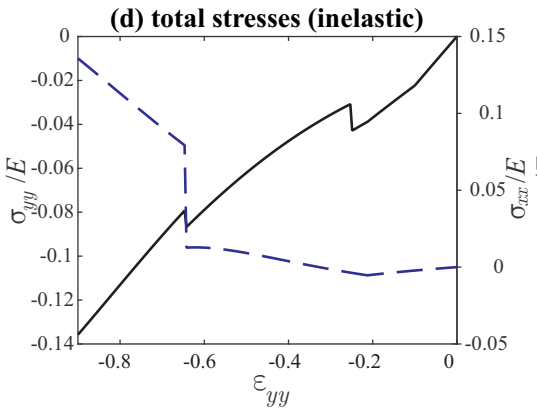

(b) bar contribution (elastic)

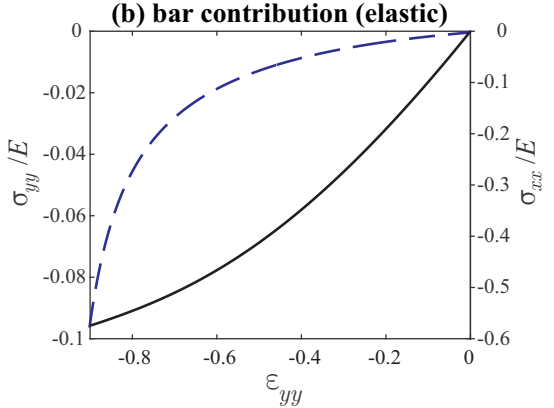

(e) bar contribution (inelastic)

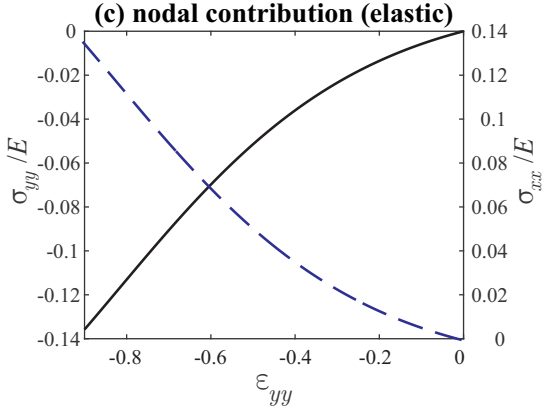

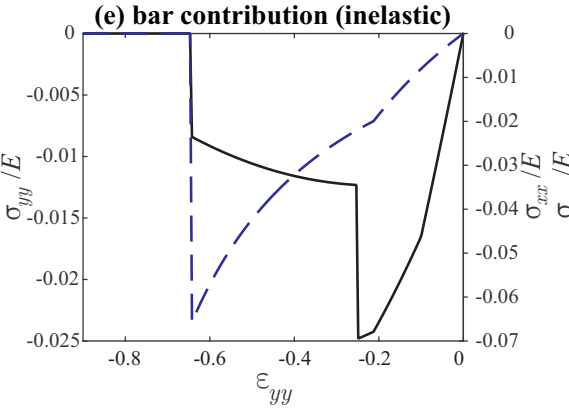

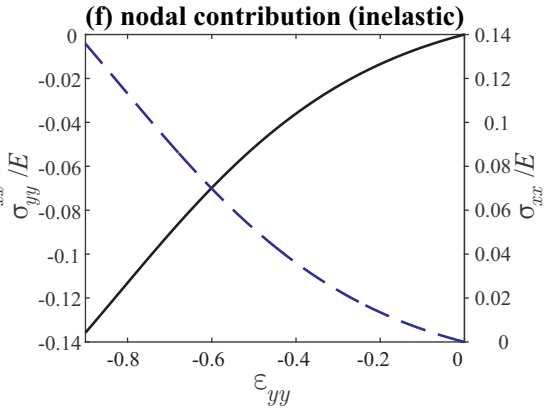

Figure 4: Cauchy stress components during uniform uniaxial compression of a periodic truss with square-type architecture, using the local continuum model with elastic nodes (with $E=K$ ) and (a-c) elastic bars or (d-e) an elastic-ideal plastic-failure model for the bars.

implying constant beam thicknesses $D$ as found in many engineering applications. For convenience, here and in the following we use

$$
\gamma=\frac{d K}{E}
$$

as a dimensionless metric for the ratio of nodal to bar stiffness, so that $\gamma=0.1$ in this example with $K=E$.

For the case of elastic bars and nodes according to (22) with $E=K$, Figs. 4(a)-(c) summarize the resulting true stress components in the compressed and transverse directions, $\sigma_{y y}$ and $\sigma_{x x}$, respectively. Also shown are the individual contributions of the bars and nodes to the total stress components. The total stresses show the expected behavior: monotonous decrease of the compressive stress $\sigma_{y y} \leq 0$ and a negative transverse stress component $\sigma_{x x} \leq 0$ (due to the constraint $F_{22}=1$ ). Interesting are the different contributions of nodes and bars: while the stresses resulting from bar stretching show the same trends as the total stresses, the nodal contribution indicates auxetic behavior (i.e., a negative Poisson effect) since $\sigma_{x x} \geq 0$ for the nodal contribution. Indeed, rigid bars connected by compliant nodes may result in auxeticity (Desmoulins et al., 2016).

As an example of inelastic behavior, Figs. 4(d)-(f) show the analogous results for elastic nodes but inelastic bars which show the elastic-plastic-failure response according to (24), a yield stress of $\tau_{0}=0.1 E$ and hardening modulus $S=0.1 E$, and with bar failure occurring at $\varepsilon_{\mathrm{cr}}=0.25$. In addition to plastic softening (resulting in lower stresses), one clearly recognizes the failure of bars indicated by the discrete stress bursts in the bar contribution to the stresses (due to their orientation, vertical bars fail before the diagonal ones, and horizontal bars do not fail for the chosen deformation path).

Of course, a simple material point calculation cannot leverage the nonlocal continuum model nor predict the response of boundary value problems, which is why we perform finite element simulations in the following sections to highlight strengths and weaknesses of the local and nonlocal continuum models.

\subsection{Elastic trusses: local continuum model}

As the following examples show, the nonlinear deformation of elastic trusses is sufficiently well captured by the local continuum model if no localization occurs (the nonlocal terms provide only minor improvements of all simulated results shown). As an example, consider the uniaxial compression of a body made of the X-braced square 
truss, which is constrained at its top and bottom and deforming freely otherwise. Fig. 5 compares the results of discrete truss calculations (shown by the wire frame are the deformed bars according to the exact, discrete solution) to those of an FE simulation using the local continuum model discussed above (shown as a solid body color-coded by deformation gradient component $F_{x x}$ ). The two shown FE meshes in Figs. 5a and Figs. 5b have only about $3.40 \%$ and $0.93 \%$, respectively, of the total number of degrees of freedom (dofs) used in the discrete lattice calculation. It becomes apparent that despite the inhomogeneous deformation, the deformed shapes of the discrete and continuum models show convincing agreement in both cases, with deviations vanishing with decreasing mesh size. As the finite element mesh is considerably more coarse than the discrete lattice resolution, fine features such as the deformation near the corners cannot be captured most accurately by the local model, as can be expected. Besides, the choice of $\gamma=1(K=10 E, d=0.1)$ again leads to auxetic behavior.

Fig. 6 shows the corresponding load-displacement curves for a total of four different FE meshes, compared to the exact solution of the discrete truss. While the initial linear behavior up to about $20 \%$ compressive strain hardly indistinguishable, differences become apparent at larger strains. Again, with decreasing mesh size the continuum results converge towards the exact solution while using less dofs. Shown on the right are the distributions of the two displacement components for the discrete lattice (right) and the continuum model with 882 dofs at a compressive strain of $25 \%$, again showing convincing agreement.

As a quantitative error metric for the above example, we show the mean relative displacement errors in Fig. 7. The definition of a comparable global error norm is challenging since the discrete and the FE representations use different meshes, so that displacements are defined on the nodes of two different grids (also, an energy error norm is little helpful due to different definitions of energy in the two models). As a simple error measure, the mean relative displacement error is computed across all nodes shared by both nodal point sets (by choosing the mesh sizes such as to achieve a maximum coincidence of nodes). Fig. 7 shows that the error increases with increasing level of deformation as well as with decreasing mesh resolution, as can be expected. The discrete reference truss contains 80 unit cells per side and a total of 25,922 dofs. Results indicate that all shown continuum simulations keep the mean relative error below $1 \%$ for the shown levels of deformation.

As a further example, Fig. 8 compares discrete and continuum results of a simulated indentation test using the same FE meshes as before (a spherical indenter of diameter $0.5 \mathrm{H}$ is modeled by an external potential (Kelchner et al., 1998)). Again, the agreement of the deformed shapes of both models is excellent despite the significantly reduced number of degrees of freedom in the continuum representation (the coarse FE discretization becomes apparent in Fig. 8(b) where the circular indentation imprint can be reproduced only approximately due to the triangular elements used for FE representation). Colors illustrate the inhomogeneous distribution of the vertical displacements.

To verify that the model is suitable for other truss architectures, Fig. 9 shows results for the triangular lattice whose unit cell was shown in Fig. 1c (results are for $\gamma=0.05$ at $23.1 \%$ vertical indentation). The discrete model contains 3,402 dofs, whereas the continuum simulation was performed with 462 dofs. As before, the agreement between the

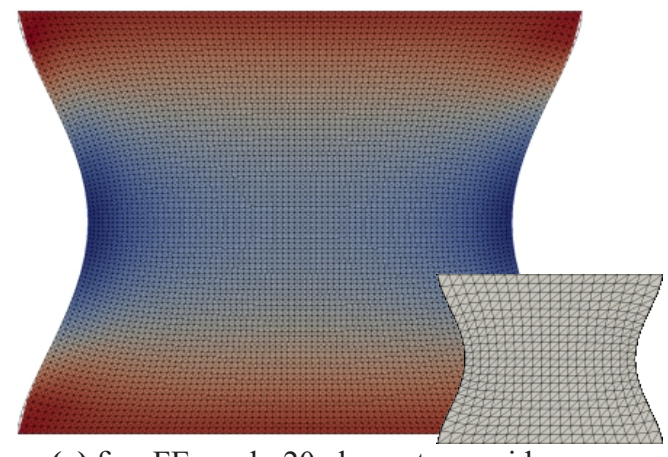

(a) fine FE mesh: 20 elements per side, 882 dofs $\approx 3.40 \%$

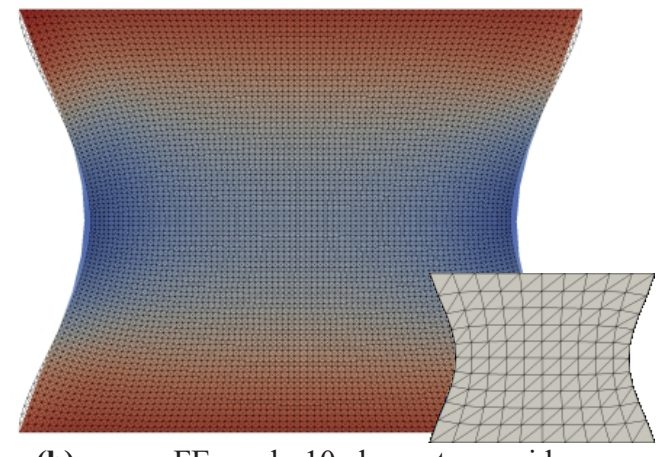

(b) coarse FE mesh: 10 elements per side, 242 dofs $\approx 0.93 \%$

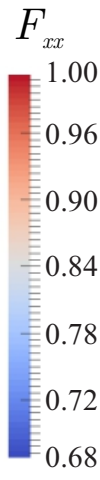

1.00

0.90

78

.68

gure 5: Square-type lattice at 25\% vertical compression (with $\gamma=1$, top and bottom are rigidly constrained, sides are free): comparison of the discrete truss simulation (80 unit cells per side, 25,922 dof; shown as the wire frame) and the local continuum model with two different FE representations (meshes are shown in gray). The color code visualizes the inhomogeneous distribution of deformation gradient component $F_{x x}$. 

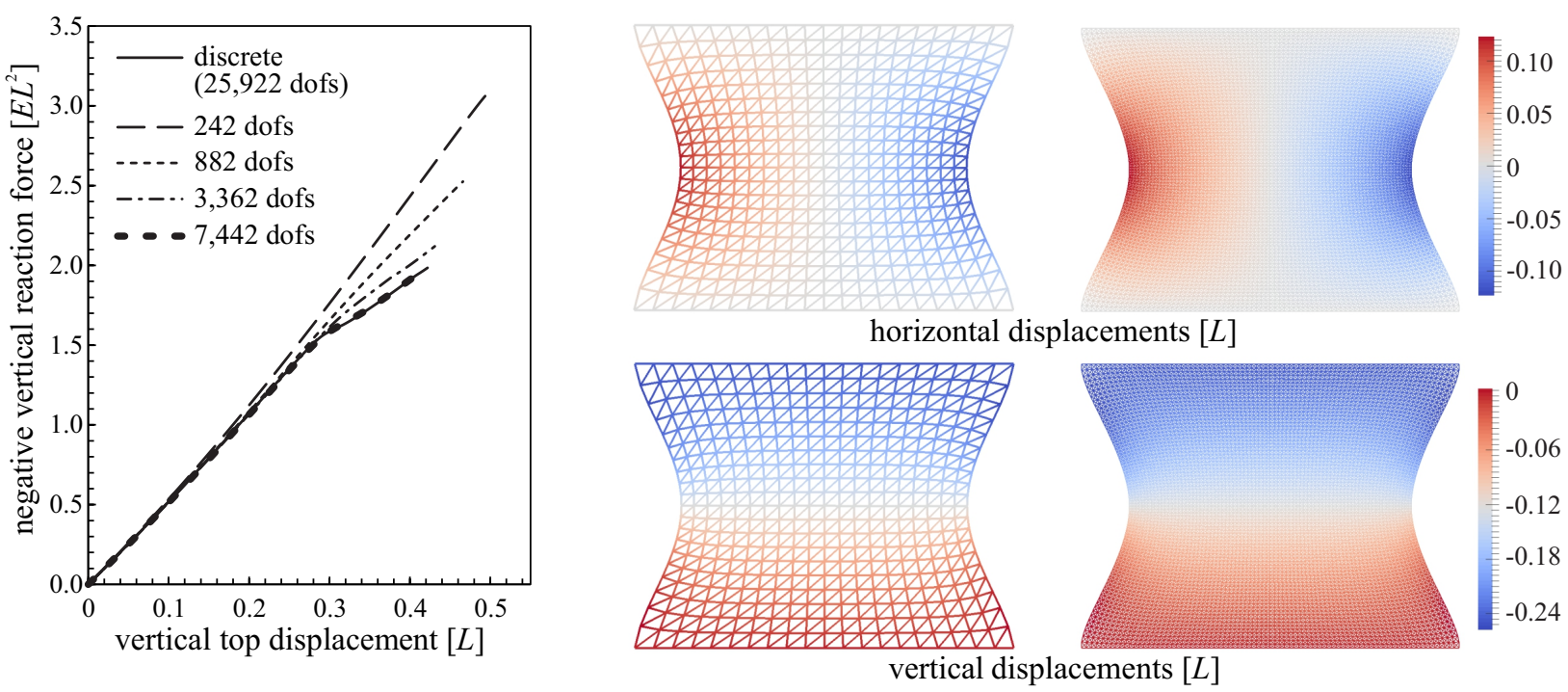

Figure 6: Results of the elastic compression test: normalized load-displacement curve comparing the exact discrete data to the local continuum model with different mesh sizes (shown are the total numbers of degrees of freedom (dofs) in the finite element meshes). Displacements fields are shown for the discrete lattice (right) and the continuum model with 882 dofs (left).

two predicted deformed shapes is excellent. Fig. 9 also shows the inhomogeneous strain distribution by visualizing the four components of the two-dimensional deformation gradient in the continuum model. Note that the choice of $\gamma=0.05$ leads to a positive Poisson effect in contrast to the above auxetic lattice response.

When considering elastic behavior (without instabilities), all of the above examples confirm convincing agreement between the local continuum model and the exact discrete model. Using the nonlocal model with the additional regularization terms cause only negligible differences in the results shown above for the local model.

\subsection{Inelastic trusses: nonlocal continuum model}

Nonlocal effects become important, e.g., when localization occurs. This can be induced at sufficiently large levels of deformation when trusses are loaded beyond the elastic limit and plastic flow may be expected (as demonstrated in Section 4.1). Specifically, in the uniaxial compression tests conducted before, localization can occur in the form of creases on the free edges of the discrete truss network, especially if the elastic parameters are chosen such that the resulting truss behavior is auxetic. We employ our nonlocal model to assess the accuracy of the continuum

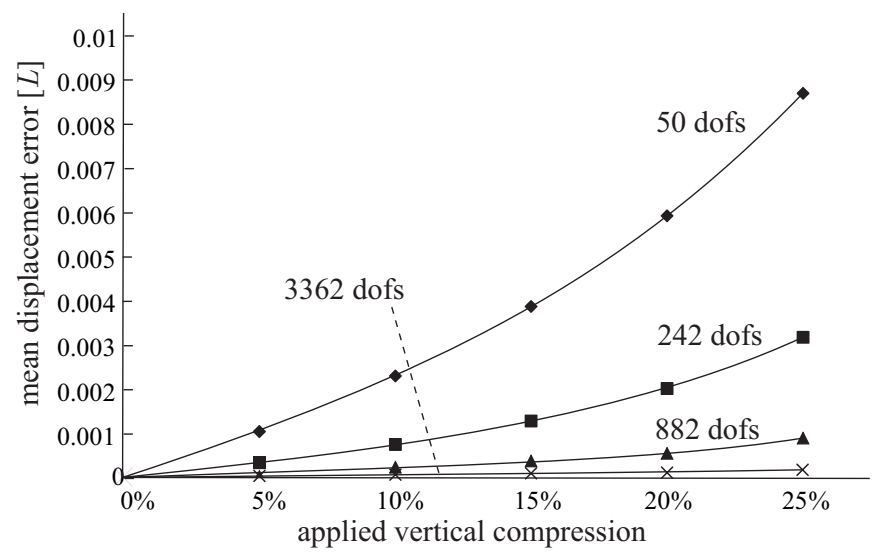

Figure 7: Mean relative displacement error vs. vertical compression for four different FE meshes used with the local continuum model (the total number of degrees of freedom in each FE mesh is indicated). 


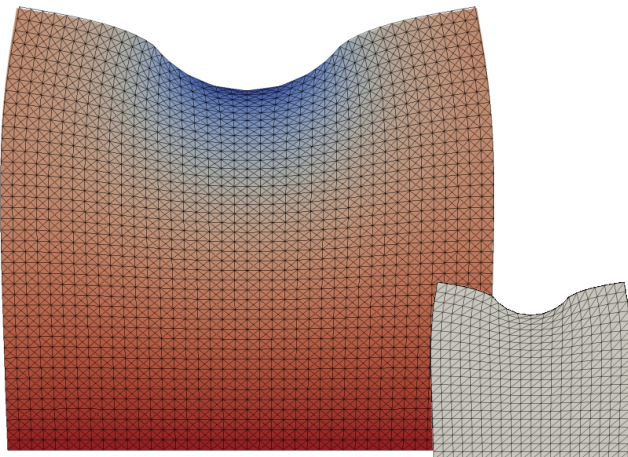

(a) fine FE mesh: 20 elements per side, 882 dofs $\approx 13.4 \%$

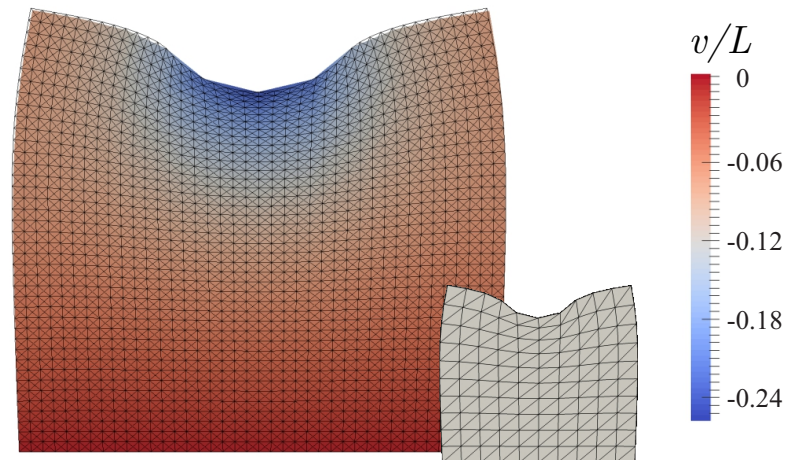

(b) coarse FE mesh: 10 elements per side, 242 dofs $\approx 3.69 \%$

Figure 8: Square-type lattice at $25 \%$ indentation depth (with $\gamma=0.1$, bottom is constrained, sides are free): comparison of the discrete truss simulation (40 unit cells per side, 6,562 dof) and the local continuum model with two different meshes (shown in gray). The color code illustrates differences in the (normalized) vertical displacement component $v$.

model's predictions in this case, using the truss plasticity model of Section 2.3 for the cases of indentation and compression. Note that the local model is included in the nonlocal model if we let the intrinsic length scale vanish (i.e., $L \rightarrow 0$ ). To produce localization in a controlled manner, we choose $\gamma=0.1$ in subsequent simulations to create auxetic behavior. It is important to point out that the discrete truss description underlying all examples here does not admit beam buckling (the same model can, in principle, be used to capture beam buckling by subdividing each bar into multiple bars connected by $180^{\circ}$-nodal connections; yet this is not considered here). The behavior may thus be viewed as characteristic for non-slender, stretching-dominated trusses with high $t / L$ ratios, see, e.g., (Meza et al., 2015). Validation of the micro-to-macro transition scheme and resulting continuum model is performed by the only fair comparison, viz. by comparing to discrete lattice calculations using the exact same bar/node constitutive assumptions. As discussed before, an extension to more complex truss descriptions is warranted and readily available within the presented techniques but not the focus here.

Fig. 10 illustrates the deformed shape of a compressed square truss at $34.4 \%$ compression as obtained from a discrete truss calculation and the nonlocal model used in an FE simulation (with $S=E / 6, \tau_{0} / E=0.01$ ). Results were computed with 21 nodes per side in the FE model, compared to 41 nodes in the discrete truss. The influence of the nonlocal terms is controlled through the unit cell side length $L$ in the continuum model. Obviously, the local model $(L \rightarrow 0)$ is not capable of reproducing the localization phenomenon despite sufficient mesh refinement, as shown in Fig. 10(d,h). The nonlocal model with $L=0.025 H$ (which is the same unit cell size as the actual bar length in the discrete truss, i.e., $L=\bar{L}=H / 40$ ) reproduces the plastic localization zone best. The magnification shows differences, among others due to the coarser mesh used in the FE simulation, but the general trend is captured surprisingly well for $L=\bar{L}$ in Fig. 10(a,e). Also, the magnified result in Fig. 10(e) is the only one that captures the expected increase in the second-order gradient near the crease.

Fig. 11 shows the load-displacement curve for the above elastic-plastic compression test, along with the stress
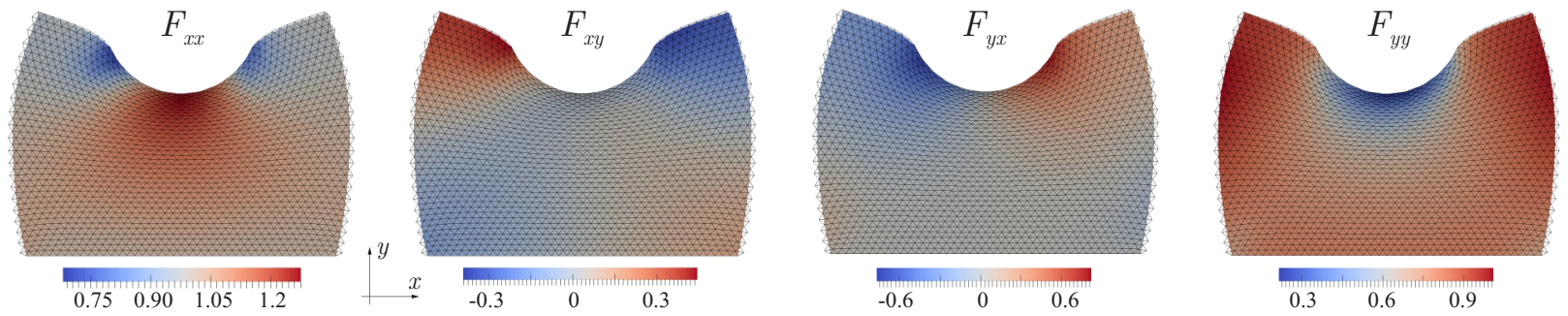

Figure 9: Indentation test using the triangular lattice and the local continuum model. The color code illustrates the deformation gradient components in the continuum model, the deformed shape of the exact discrete truss simulation is shown by the wire frame for comparison. 


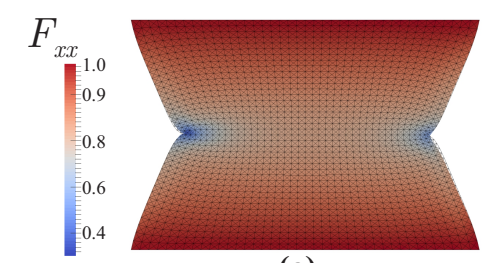

(a)

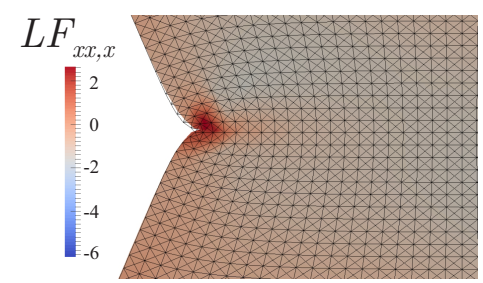

(e)

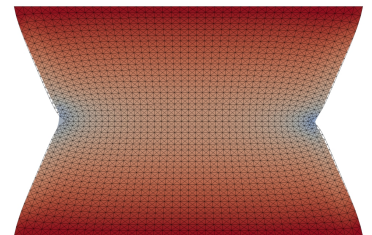

(b)

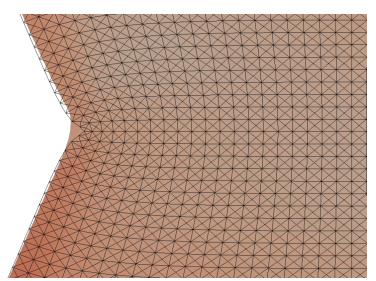

(f)

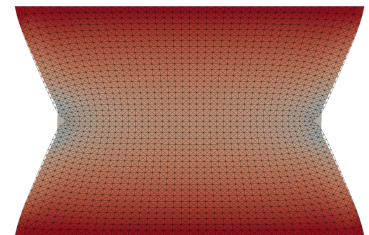

(c)

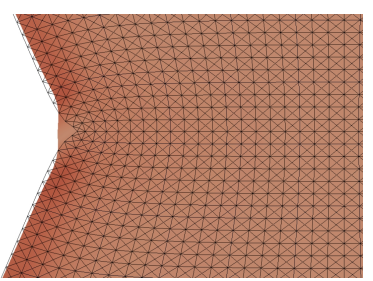

(g)

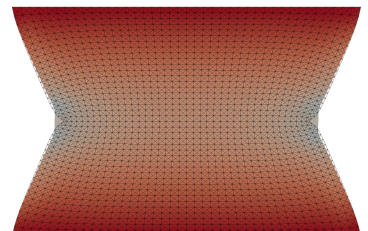

(d)

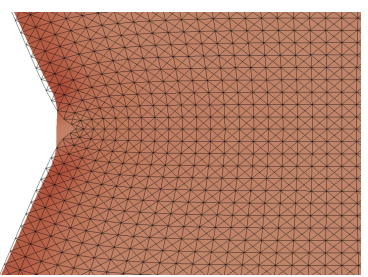

(h)

Figure 10: Simulated compression test using the nonlocal continuum model in comparison to the discrete truss calculation. The color code shows the horizontal deformation gradient component $F_{x x}$ and the second-order gradient $F_{x x, x}$ (normalized by $1 / L$ ); the bottom figures are magnifications of the left crazes shown directly above. The nonlocal length scale parameter $L / H$ (i.e., the size of the unit cell in the nonlocal continuum model normalized by the truss side length) is $(\mathrm{a}, \mathrm{e}) 2.5 \cdot 10^{-2},(\mathrm{~b}, \mathrm{f}) 10^{-2},(\mathrm{c}, \mathrm{g}) 10^{-3},(\mathrm{~d}, \mathrm{~h}) 0$. Therefore, cases $(\mathrm{d}, \mathrm{h})$ correspond to the local model $(L \rightarrow 0)$.

distribution within the discrete and FE representations. The load-displacement curve impressively demonstrates that only the choice $L=\bar{L}=H / 40$ reproduces the exact, discrete results. Most importantly, we see that choices of both $L>\bar{L}$ and $L<\bar{L}$ lead to incorrect FE results, while the local model is far from the exact solution beyond $20 \%$ of applied compression. We note that the curves terminate at different levels of applied compression due to numerical instability (using a Newton-Raphson solver with identical load increments). The local model fails early on, as does the nonlocal model with small values of the intrinsic length $\bar{L}$. Only the choice $L=\bar{L}$ leads to stable results that agree almost exactly with the discrete calculation up to $50 \%$ compression. The stress distributions, shown in Fig. 11(b,c), admit a qualitative comparison. We note that a quantitative comparison is difficult since one component of the continuum stress tensor is compared to the discrete axial bar stresses.

Finally, Fig. 12 shows the results of a simulated indentation test, forcing a spherical indenter of diameter $0.5 H$ into an elastic-plastic square truss (with $S=E / 6, \tau_{0} / E=0.01$ ), shown at an indentation depth of $40 \%$ of the initial truss height. For the simulation we used five different values of the internal length $L$ in the nonlocal model. The discrete truss has unit cell length $\bar{L}=H / 30$. Apparently, the best agreement of the deformed shape in the localized region is again obtained with the exact choice $L=\bar{L}$; see cases $(\mathrm{c}, \mathrm{h})$. Largest discrepancies are observed for the extremal cases of $L=10^{-1} H$ and $L=10^{-3} H$. Note that, in addition to producing incorrect results, large choices of $L$ are also theoretically questionable since they break the underlying separation-of-scales assumption.

Both the indentation and compression examples have shown that the best agreement with the exact, discrete results is achieved when the unit cell size in the nonlocal model is chosen to coincide with the actual, physical size of the unit cell in the discrete truss. Of course, we can only show representative examples here; yet, all our examples not shown here in full detail have confirmed this conclusion. While not surprising, this indicates that the nonlocal continuum model accurately reflects size effects in the truss network. Interestingly, the examples shown in Figs. 10 to 12 demonstrate that features (such as localization zones) on the same order as the underlying discrete unit cell are represented well by the nonlocal continuum model (not by the local model). This indicates that, although the continuum model is based on a representative unit cell and we thus started out by assuming a clear separation of scales between macroscopic body and underlying discrete unit cell, the nonlocal continuum model also provides promising results in cases where this assumption breaks down. Of course, this generally requires sufficient mesh resolution in regions showing fine features. Yet, the latter can be supplied by mesh adaptivity (this, however, goes beyond the scope of the present investigation). Also, since the FE discretization is chosen such that elements are always larger than the discrete unit cell ${ }^{4}$ and $C^{1}$-continuous elements are being used, the deformation gradient is guaranteed to vary

\footnotetext{
${ }^{4}$ The main purpose of the presented methods was to enable an efficient FE representation that does not require resolution at the level of individual truss features.
} 

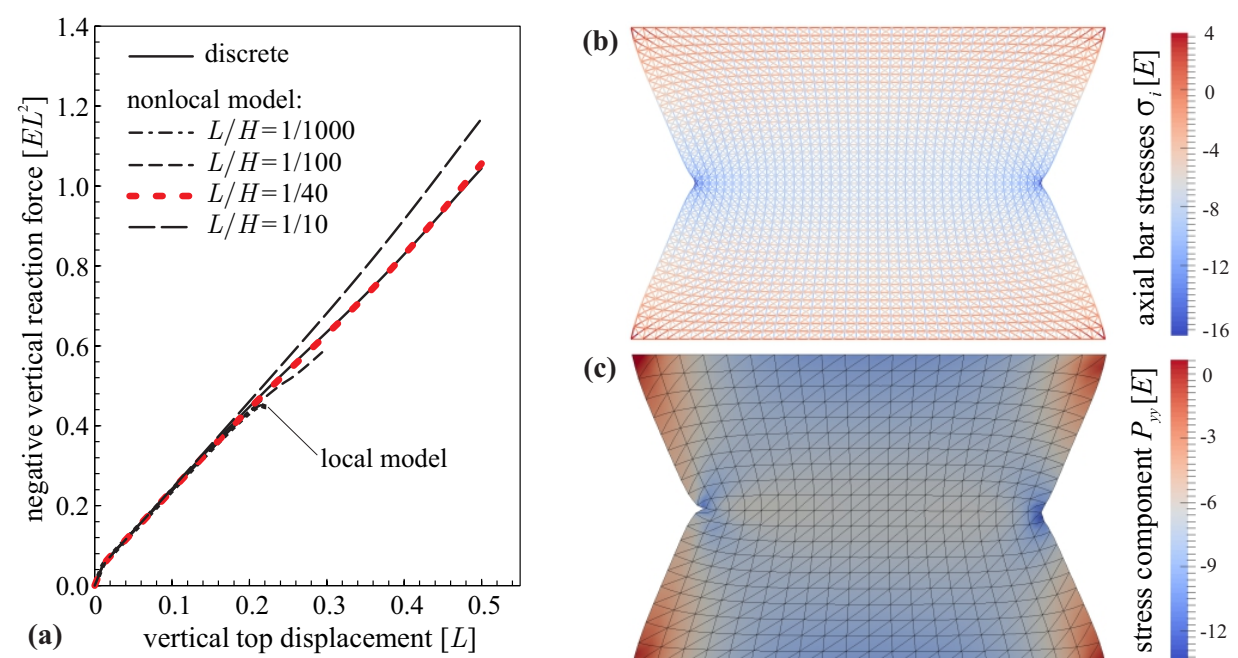

(c)

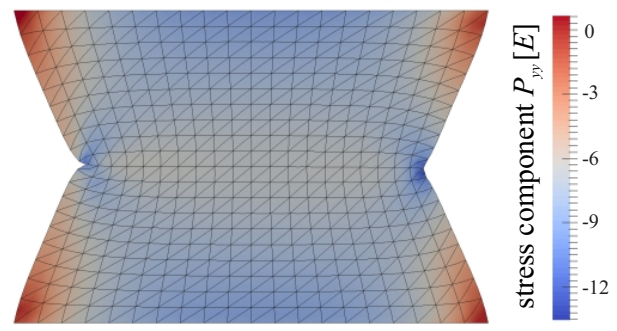

Figure 11: (a) Force-displacement curves for the compression test of Fig. 10: comparison of the exact discrete results to data obtained from the nonlocal and local models with different meshes. Further shown is a comparison of (b) the axial bar stress distribution in the discrete case and (c) the distribution of stress component $P_{y y}$ obtained from the nonlocal continuum model.

at most linearly at the level of the RUC (except across element boundaries), as required by the separation of scales (see Section 2.2).

\section{Conclusions}

We have shown that the quasistatic response of periodic discrete networks can be efficiently modeled by continuum models which apply the traditional or extended Cauchy-Born rule to a representative unit cell of the discrete truss. These two variations define a local and nonlocal continuum model, respectively. We specifically considered lattices whose deformation can be described based on translational degrees of freedom only (while accounting for angle

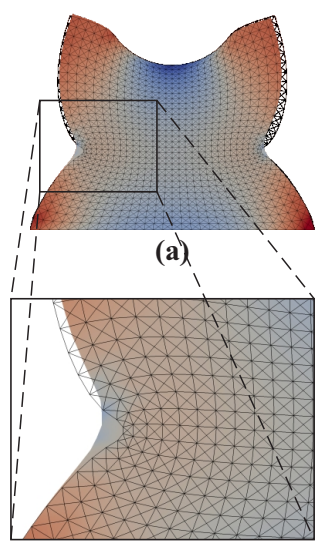

(f)

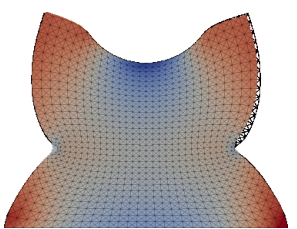

(b)

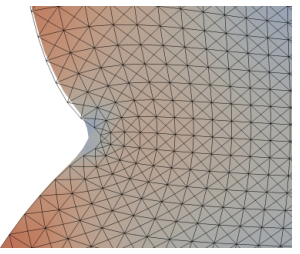

(g)

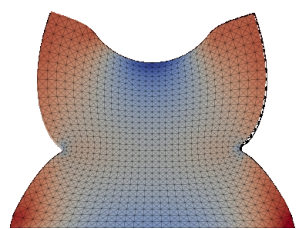

(c)

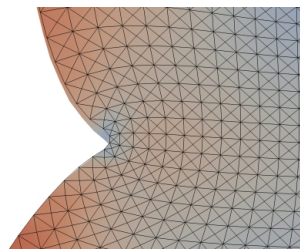

(h)

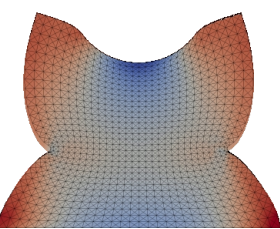

(d)

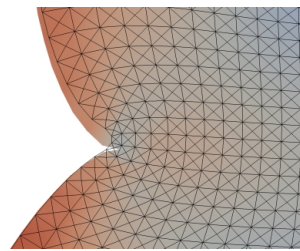

(i)

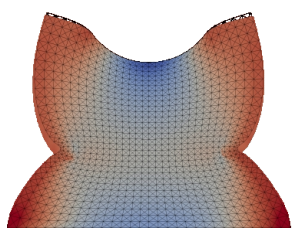

(e)

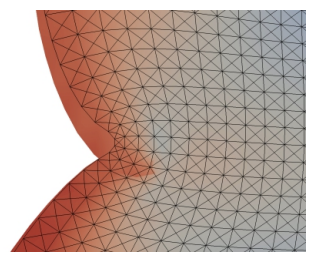

(j)

$\begin{array}{cccccc}-0.05 & -0.04 & -0.03 & -0.01 & 0 & 0.02\end{array} P_{y y} / E$

Figure 12: Simulated indentation test using the nonlocal continuum model in comparison to the discrete truss calculation. The color code shows the vertical component $P_{y y}$ of the first Piola-Kirchhoff stress tensor; the bottom figures are magnified versions of the highlighted region. The nonlocal length ratio $L / H$ (i.e., the size of the RUC in the nonlocal continuum model normalized by the truss side length) is (a,f) $10^{-1},(\mathrm{~b}, \mathrm{~g}) 5 \cdot 10^{-2},(\mathrm{c}, \mathrm{h})$ $3.3 \cdot 10^{-2},(\mathrm{~d}, \mathrm{i}) 2 \cdot 10^{-2},(\mathrm{e}, \mathrm{j}) 10^{-3}$. 
changes). A prototypical example is the class of stretching-dominated truss lattices undergoing finite deformations, whose nodes possess a finite elastic stiffness (thus mimicking experimental findings of nodal behavior in small-scale truss networks). For numerical compression and indentation examples, we showed that the deformation response of elastic trusses is captured well by the local continuum model at significantly lower computational costs achieved by a coarse finite element mesh resolution. Large deformation or plasticity in truss lattices, by contrast, can produce localization zones (here, shown for the example of elastic-plastic auxetic lattices). This behavior is captured well only by the nonlocal continuum model, and best results have been achieved if the unit cell size in the nonlocal model coincides with the actual size of the discrete truss unit cell. This observation also applies to the shown examples in which a separation of scales between element size and unit cell size no longer holds. The micro-to-macro transition scheme discussed here can be extended to arbitrary discrete networks as long as the constitutive response of individual lattice members can be described by translational degrees of freedom (including, e.g., truss networks, atomistic crystals, or granular media). There is clearly room to improve upon the underlying discrete constitutive models, e.g., through detailed FE models (see, e.g., Meza et al. (2015)) or through experiments revealing more complex bar and nodal constitutive behavior.

\section{Acknowledgment}

Support from the National Science Foundation (NSF) through CAREER award CMMI-1254424 is gratefully acknowledged.

\section{References}

Amelang, J. S., Venturini, G. N., Kochmann, D. M., 2015. Summation rules for a fully nonlocal energy-based quasicontinuum method. Journal of the Mechanics and Physics of Solids 82, 378-413.

URL http://www.sciencedirect.com/science/article/pii/S0022509615000630

Askes, H., Metrikine, A., 2005. Higher-order continua derived from discrete media: continualisation aspects and boundary conditions. International Journal of Solids and Structures 42 (1), 187-202.

Bardenhagen, S., Triantafyllidis, N., 1994. Derivation of higher order gradient continuum theories in 2, 3-d non-linear elasticity from periodic lattice models. Journal of the Mechanics and Physics of Solids 42 (1), 111-139.

Beex, L., Peerlings, R., Geers, M., 2014. Central summation in the quasicontinuum method. Journal of the Mechanics and Physics of Solids 70 (0), $242-261$.

URL http://www.sciencedirect.com/science/article/pii/S0022509614001100

Carstensen, C., Hackl, K., Mielke, A., 2002. Non-convex potentials and microstructures in finite-strain plasticity. Proceedings of the Royal Society of London A: Mathematical, Physical and Engineering Sciences 458 (2018), 299-317.

URL http://rspa.royalsocietypublishing.org/content/458/2018/299

De Borst, R., Sluys, L., Muhlhaus, H.-B., Pamin, J., 1993. Fundamental issues in finite element analyses of localization of deformation. Engineering computations $10(2), 99-121$.

Deshpande, V., Fleck, N., Ashby, M., 2001a. Effective properties of the octet-truss lattice material. Journal of the Mechanics and Physics of Solids 49 (8), 1747-1769.

URL http://www.sciencedirect.com/science/article/pii/S0022509601000102

Deshpande, V. S., Ashby, M. F., Fleck, N. A., 2001b. Foam topology: bending versus stretching dominated architectures. Acta Mater. 49, 10351040 .

Desmoulins, A., Zelhofer, A. J., Kochmann, D. M., 2016. Auxeticity in truss networks and the role of bending versus stretching deformation. Smart Materials and Structures 25 (5), 054003.

URL http: //stacks .iop.org/0964-1726/25/i=5/a=054003

Dow, J., Huyer, S., 1989. Continuum models of space station structures. Journal of Aerospace Engineering 2 (4), $220-238$.

Ericksen, J., 2008. On the cauchyborn rule. Mathematics and Mechanics of Solids 13 (3-4), 199-220.

URL http://mms. sagepub.com/content/13/3-4/199. abstract

Eringen, A., 1965. Linear theory of micropolar elasticity. Tech. rep., DTIC Document.

Eringen, A., 1966. Linear theory of micropolar elasticity. Indiana Univ. Math. J. 15, 909-923.

Fan, H., Jin, F., Fang, D., 2009. Nonlinear mechanical properties of lattice truss materials. Materials \& Design 30 (3), $511-517$. URL http://www.sciencedirect.com/science/article/pii/S0261306908002380

Feyel, F., Chaboche, J.-L., 2000. Fe2 multiscale approach for modelling the elastoviscoplastic behaviour of long fibre sic/ti composite materials. Computer Methods in Applied Mechanics and Engineering 183 (3-4), 309 - 330.

URL http://www.sciencedirect.com/science/article/pii/S0045782599002248

Fleck, N., 2001. Multi-axial strength of metallic foams and lattice materials. Journal of the JSMS: Materials Science for the 21st Century A, 336-340.

Fleck, N., Deshpande, V. S., Ashby, M. F., 2010a. Micro-architectured materials: past, present and future. Proc. Roy. Soc. A 466, $2495-516$. 
Fleck, N. A., Deshpande, V. S., Ashby, M. F., 2010b. Micro-architectured materials: past, present and future. Proceedings of the Royal Society of London A: Mathematical, Physical and Engineering Sciences 466 (2121), 2495-2516.

Fleck, N. A., Qiu, X., 2007. The damage tolerance of elastic-brittle, two-dimensional isotropic lattices. J. Mech. Phys. Solids 55, $562-588$.

Gibson, L. J., Ashby, M. F., 1999. Cellular Solids: Structure and Properties. Cambridge University Press.

Gonella, S., Ruzzene, M., 2008. Homogenization of vibrating periodic lattice structures. Applied Mathematical Modelling 32 (4), 459-482.

Hu, J., Park, T., 2013. Continuum models for the plastic deformation of octet-truss lattice materials under multiaxial loading. Journal of Engineering Materials and Technology 135 (2), 021004.

Hutchinson, R., Fleck, N. A., 2006. The structural performance of the periodic truss. J. Mech. Phys. Solids, 756-82.

Jacobsen, A. J., Barvosa-Carter, W., Nutt, S., 2007. Micro-scale truss structures formed from self-propagating photopolymer waveguides. Adv. Mater. 19, 3892-3896.

Kelchner, C. L., Plimpton, S. J., Hamilton, J. C., 1998. Dislocation nucleation and defect structure during surface indentation. Phys. Rev. B 58, $11085-11088$

Kirkwood, J., 1939. The skeletal modes of vibration of long chain molecules. The Journal of Chemical Physics 7 (7), 506-509.

Kochmann, D. M., Amelang, J. S., 2016. The quasicontinuum method: theory and applications. In: Weinberger, C. R., Tucker, G. J. (Eds.), Multiscale Materials Modeling for Nanomechanics. Springer, New York, pp. 159-193.

Koiter, W. T., 1964. Couple stresses in the theory of elasticity, I \& II. Proc. K. Ned. Akad. Wet. (B) 67, 1744.

Krödel, S., Delpero, T., Bergamini, A., Ermanni, P., Kochmann, D. M., 2014. 3D auxetic microlattices with independently-controllable acoustic band gaps and quasi-static elastic moduli. Advanced Engineering Materials 16, 357-363. URL http: //onlinelibrary.wiley.com/doi/10.1002/adem.201300264/abstract

Kumar, P., 2011. Dynamic response of structural materials to shock loading. Ph.D. thesis, The University of Rhode Island. URL http://digitalcommons .uri.edu/dissertations/AAI3488416

Kumar, R., McDowell, D., 2004. Generalized continuum modeling of 2-d periodic cellular solids. International Journal of Solids and Structures 41 (26), 7399-7422

McCallen, D., Romstad, K., 1988. A continuum model for the nonlinear analysis of beam-like lattice structures. Computers \& structures 29 (2), 177-197.

McCallen, D., Romstad, K., 1990. A continuum model for lattice structures with geometric and material nonlinearities. Computers \& Structures 37 (5), 795-822

Metrikine, A., Askes, H., 2006. An isotropic dynamically consistent gradient elasticity model derived from a 2d lattice. Philosophical Magazine $86(21-22), 3259-3286$

Meza, L. R., Das, S., Greer, J. R., 2014. Strong, lightweight, and recoverable three-dimensional ceramic nanolattices. Science 345 (6202), $1322-$ 1326. URL http://www.sciencemag.org/content/345/6202/1322.abstract

Meza, L. R., Zelhofer, A. J., Clarke, N., Mateos, A. J., Kochmann, D. M., Greer, J. R., 2015. Resilient 3d hierarchical architected metamaterials. Proceedings of the National Academy of Sciences 112 (37), 11502-11507. URL http: //www . pnas . org/content/112/37/11502 . abstract

Miehe, C., Schröder, J., Becker, M., 2002. Computational homogenization analysis in finite elasticity: material and structural instabilities on the micro- and macro-scales of periodic composites and their interaction. Computer Methods in Applied Mechanics and Engineering 191 (44), 4971-5005.

URL http://www.sciencedirect.com/science/article/pii/S0045782502003912

Mielke, A., 2002. Geometry, Mechanics, and Dynamics. Springer New York, New York, NY, Ch. Finite Elastoplasticity Lie Groups and Geodesics on $\operatorname{SL}(\mathrm{d})$, pp. 61-90.

URL http://dx.doi.org/10.1007/0-387-21791-6_2

Mindlin, R., 1965. Second gradient of strain and surface-tension in linear elasticity. International Journal of Solids and Structures 1 (4), 417-438.

Montemayor, L., 2015. Fabrication, characterization, and deformation of 3d structural meta-materials. Ph.D. thesis, California Institute of Technology.

Necib, B., Sun, C., 1989. Analysis of truss beams using a high order timoshenko beam finite element. Journal of sound and vibration 130 (1), 149-159.

Noor, A., Andersen, C., 1979. Analysis of beam-like lattice trusses. Computer Methods in Applied Mechanics and Engineering 20 (1), 53-70.

Odegard, G., Gates, T., Nicholson, L., Wise, K., 2002. Equivalent-continuum modeling of nano-structured materials. Composites Science and Technology 62 (14), 1869-1880.

Ortiz, M., Stainier, L., 1999. The variational formulation of viscoplastic constitutive updates. Computer Methods in Applied Mechanics and Engineering 171 (3-4), 419 - 444.

URL http://www.sciencedirect.com/science/article/pii/S0045782598002199

Pal, R. K., Ruzzene, M., Rimoli, J. J., 2016. A continuum model for nonlinear lattices under large deformations. International Journal of Solids and Structures $96,300-319$.

URL http://www.sciencedirect.com/science/article/pii/S002076831630107X

Papanicolopulos, S.-A., Zervos, A., Vardoulakis, I., 2009. A three-dimensional c1 finite element for gradient elasticity. International Journal for Numerical Methods in Engineering 77 (10), 1396-1415.

URL http: //dx.doi.org/10.1002/nme.2449

Queheillalt, D., Wadley, H., 2005. Cellular metal lattices with hollow trusses. Acta Materialia 53 (2), $303-313$.

URL http: //www.sciencedirect.com/science/article/pii/S1359645404005671

Reddy, C., Rajendran, S., Liew, K., 2005. Equivalent continuum modeling of graphene sheets. International Journal of Nanoscience 4 (04), 631636.

Romijn, N. E. R., Fleck, N. A., 2007. The fracture toughness of planar lattices: Imperfection sensitivity. Journal of the Mechanics and Physics of Solids 55 (12), 2538 - 2564. 
URL http: //www.sciencedirect.com/science/article/pii/S002250960700097X

Rudraraju, S., der Ven, A. V., Garikipati, K., 2014. Three-dimensional isogeometric solutions to general boundary value problems of toupins gradient elasticity theory at finite strains. Computer Methods in Applied Mechanics and Engineering 278, 705 - 728.

URL http: //www.sciencedirect.com/science/article/pii/S0045782514002011

Ruzzene, M., Scarpa, F., 2005. Directional and band-gap behavior of periodic auxetic lattices. physica status solidi (b) 242, 665-680. URL http://dx.doi.org/10.1002/pssb. 200460385

Schaedler, T. A., Jacobsen, A. J., Torrents, A., Sorensen, A. E., Lian, J., Greer, J. R., Valdevit, L., Carter, W. B., 2011a. Ultralight metallic microlattices. Science 334 (6058), 962-965.

URL http: //www. sciencemag.org/content/334/6058/962.abstract

Schaedler, T. A., Jacobsen, A. J., Torrents, A., Sorensen, A. E., Lian, J., Greer, J. R., Valdevit, L., Carter, W. B., 2011b. Ultralight metallic microlattices. Science 334 (6058), 962-965.

URL http: //www . sciencemag.org/content/334/6058/962.abstract

Shokrieh, M., Rafiee, R., 2010. Prediction of youngs modulus of graphene sheets and carbon nanotubes using nanoscale continuum mechanics approach. Materials \& Design 31 (2), 790-795.

Sun, Y., Liew, K., 2008. The buckling of single-walled carbon nanotubes upon bending: the higher order gradient continuum and mesh-free method. Computer Methods in Applied Mechanics and Engineering 197 (33), 3001-3013.

Sunyk, R., Steinmann, P., 2003. On higher gradients in continuum-atomistic modelling. International Journal of Solids and Structures 40 (24), 6877-6896.

Symons, D. D., Fleck, N. A., 2008. The imperfection sensitivity of isotropic two-dimensional elastic lattices. J. Appl. Mech. 75, 051011.

Tadmor, E., Ortiz, M., Phillips, R., June 1996. Quasicontinuum analysis of defects in solids. Philos. Mag. A 73 (6), $1529-1563$.

Tadmor, E., Smith, G., Bernstein, N., Kaxiras, E., 1999. Mixed finite element and atomistic formulation for complex crystals. Physical Review B 59 (1), 235.

Torrents, A., Schaedler, T. A., Jacobsen, A. J., Carter, W. B., Valdevit, L., 2012. Characterization of nickel-based microlattice materials with structural hierarchy from the nanometer to the millimeter scale. Acta Mater. 60, 3511-3523.

Toupin, R. A., 1962. Elastic materials with couple-stresses. Archive for Rational Mechanics and Analysis 11 (1), $385-414$. URL http://dx.doi.org/10.1007/BF00253945

Toupin, R. A., 1964. Theories of elasticity with couple-stress. Archive for Rational Mechanics and Analysis 17 (2), $85-112$. URL http://dx.doi.org/10.1007/BF00253050

Triantafyllidis, N., Bardenhagen, S., 1993. On higher order gradient continuum theories in 1-d nonlinear elasticity, derivation from and comparison to the corresponding discrete models. Journal of Elasticity 33 (3), 259-293.

Usik, L., 1994. Equivalent continuum models of large platelike lattice structures. International journal of solids and structures 31 (4), $457-467$.

Valdevit, L., Godfrey, S. W., Schaedler, T. A., Jacobsen, A. J., Carter, W. B., 2013. Compressive strength of hollow microlattices: Experimental characterization, modeling, and optimal design. J. Mater. Res. 28, 2461-2473.

Wadley, H., 2006. Multifunctional periodic cellular metals. Philosophical Transactions of the Royal Society A 364 (1838), 31-68. URL http://rsta.royalsocietypublishing.org/content/364/1838/31.abstract

Wadley, H., Dharmasena, K., Chen, Y., Dudt, P., Knight, D., Charette, R., Kiddy, K., 2008. Compressive response of multilayered pyramidal lattices during underwater shock loading. International Journal of Impact Engineering 35 (9), 1102-1114. URL http://www.sciencedirect.com/science/article/pii/S0734743X07000887

Wang, Y., Cuitiño, A., 2000. Three-dimensional nonlinear open-cell foams with large deformations. Journal of the Mechanics and Physics of Solids $48(5), 961-988$.

Wu, J.-S., Chen, J.-M., 1994. Dynamic analysis of spatial beam-like lattice girders. Computers \& structures 53 (4), 961-981.

Zhang, H., Wu, J., Fu, Z., 2010. Extended multiscale finite element method for elasto-plastic analysis of $2 \mathrm{~d}$ periodic lattice truss materials. Computational Mechanics 45 (6), 623-635.

Zheng, X., Lee, H., Weisgraber, T. H., Shusteff, M., DeOtte, J., Duoss, E. B., Kuntz, J. D., Biener, M. M., Ge, Q., Jackson, J. A., Kucheyev, S. O., Fang, N. X., Spadaccini, C. M., 2014. Ultralight, ultrastiff mechanical metamaterials. Science 344, $1373-7$.

\section{Appendix A. Shape function derivative calculation}

Consider an isoparametric finite element in two dimensions with local coordinates $(\xi, \eta)$, global coordinates $(x, y)$ and the isoparametric mappings $x=\sum_{a} x^{a} N^{a}(\xi, \eta)$ and $y=\sum_{a} y^{a} N^{a}(\xi, \eta)$ in 2D. Application of the inverse function theorem to each shape function leads to

$$
\left(\begin{array}{c}
N_{, \xi} \\
N_{, \eta}
\end{array}\right)=\boldsymbol{J}\left(\begin{array}{c}
N_{, x} \\
N_{, y}
\end{array}\right) \quad \text { with } \quad \boldsymbol{J}=\left(\begin{array}{cc}
x_{, \xi} & y_{, \xi} \\
x_{, \eta} & y_{, \eta}
\end{array}\right) \quad \text { so that } \quad\left(\begin{array}{c}
N_{, x} \\
N_{, y}
\end{array}\right)=\boldsymbol{J}^{-1}\left(\begin{array}{c}
N_{, \xi} \\
N_{, \eta}
\end{array}\right)
$$

with the Jacobian matrix $\boldsymbol{J}$. Similarly, we expand the second derivatives as

$$
\left(\begin{array}{c}
N_{, \xi \xi} \\
N_{, \xi \eta} \\
N_{, \eta \eta}
\end{array}\right)=\boldsymbol{J}_{1}\left(\begin{array}{c}
N_{, x} \\
N_{, y}
\end{array}\right)+\boldsymbol{J}_{2}\left(\begin{array}{c}
N_{, x x} \\
N_{, x y} \\
N_{, y y}
\end{array}\right)=\boldsymbol{J}_{1} \boldsymbol{J}^{-1}\left(\begin{array}{c}
N_{, \xi} \\
N_{, \eta}
\end{array}\right)+\boldsymbol{J}_{2}\left(\begin{array}{c}
N_{, x x} \\
N_{, x y} \\
N_{, y y}
\end{array}\right)
$$


with matrices

$$
\boldsymbol{J}_{1}=\left(\begin{array}{cc}
x_{, \xi \xi} & y_{, \xi \xi} \\
x_{, \xi \eta} & y_{, \xi \eta} \\
x_{, \eta \eta} & y_{, \eta \eta}
\end{array}\right), \quad \boldsymbol{J}_{2}=\left(\begin{array}{ccc}
\left(x_{, \xi}\right)^{2} & 2 x_{, \xi} y_{, \xi} & \left(y_{, \xi}\right)^{2} \\
x_{, \xi} x_{, \eta} & \left(x_{, \xi} y_{, \eta}+x_{, \eta} y_{, \xi}\right) & y_{, \xi} y_{, \eta} \\
\left(x_{, \eta}\right)^{2} & 2 x_{, \eta} y_{, \eta} & \left(y_{, \eta}\right)^{2}
\end{array}\right)
$$

Altogether, we arrive at

$$
\left(\begin{array}{c}
N_{, x x} \\
N_{, x y} \\
N_{, y y}
\end{array}\right)=\boldsymbol{J}_{2}^{-1}\left[\left(\begin{array}{c}
N_{, \xi \xi} \\
N_{, \xi \eta} \\
N_{, \eta \eta}
\end{array}\right)-\boldsymbol{J}_{1} \boldsymbol{J}^{-1}\left(\begin{array}{c}
N_{, \xi} \\
N_{, \eta}
\end{array}\right)\right] \text {. }
$$

(A.1) and (A.4) are used to compute the first and second shape function derivatives for an isoparametric 2D element.

\section{Appendix B. Finite element formulation of the 2-node junction element}

Consider a $2 \mathrm{D}$ truss junction whose state of deformation is described by the nodal displacements $\boldsymbol{U}=\left\{\boldsymbol{u}_{0}, \boldsymbol{u}_{1}, \boldsymbol{u}_{2}\right\}$ of the three nodes, i.e., the junction node (labeled 0 ) and the two adjacent nodes (labeled 1 and 2) which are attached through bars. The deformed node locations simply follow as $\boldsymbol{x}_{\alpha}=\boldsymbol{u}_{\alpha}+\boldsymbol{X}_{\alpha}$. With $\theta$ the deformed angle subtended by the two bars at the junction, the energy density of the 2-bar junction element is assumed to have the general form $W=W(\theta)$. We define the distance vectors as $\boldsymbol{l}_{v}=\boldsymbol{x}_{v}-\boldsymbol{x}_{0}$ (for $\left.v=1,2\right)$ and $\hat{\boldsymbol{l}}_{v}=\boldsymbol{l}_{v} /\left|\boldsymbol{l}_{v}\right|$ (for clarity we write the components of $\boldsymbol{l}_{v}$ as $l_{i}^{v}$ where $\left.i=1,2\right)$. With the abbreviations $W^{\prime}=\partial W / \partial \theta$ and $W^{\prime \prime}=\partial^{2} W / \partial \theta^{2}$, the complete element force vector conjugate to $\boldsymbol{U}$ follows as

$$
\boldsymbol{F}=\frac{\partial W}{\partial \boldsymbol{U}}=\frac{W^{\prime}(\theta)}{s}\left(\begin{array}{c}
-\left[\left(\hat{\boldsymbol{l}}_{1} \otimes \hat{\boldsymbol{l}}_{1}-\boldsymbol{I}\right) \hat{\boldsymbol{l}}_{2} / l_{1}+\left(\hat{\boldsymbol{l}}_{2} \otimes \hat{\boldsymbol{l}}_{2}-\boldsymbol{I}\right) \hat{\boldsymbol{l}}_{1} / l_{2}\right] \\
\left(\hat{\boldsymbol{l}}_{1} \otimes \hat{\boldsymbol{l}}_{1}-\boldsymbol{I}\right) \hat{\boldsymbol{l}}_{2} / l_{1} \\
\left(\hat{\boldsymbol{l}}_{2} \otimes \hat{\boldsymbol{l}}_{2}-\boldsymbol{I}\right) \hat{\boldsymbol{l}}_{1} / l_{2}
\end{array}\right), \quad s=\sqrt{1-\left(\hat{\boldsymbol{l}}_{1} \cdot \hat{\boldsymbol{l}}_{2}\right)^{2}}=|\sin \theta| .
$$

The incremental element stiffness matrix is obtained by differentiation. Due to complexity, we present the components $K_{c b}^{i j}=\partial F_{c}^{i} / \partial u_{b}^{j}$ for the five particular cases that may be considered individually (no index summations are implied in the following except where explicitly stated). First, if $\alpha$ and $\beta$ denote exterior nodes $(\alpha, \beta=1,2)$ and $\alpha \neq \beta$, we have (using Einstein's summation convention over repeated Roman indices in two dimensions)

$$
K_{i j}^{\alpha \beta}=-\frac{W^{\prime}(\theta)}{s l_{\alpha} l_{\beta}}\left(\hat{l}_{k}^{\alpha} \hat{l}_{i}^{\alpha}-\delta_{i k}\right)\left(\hat{l}_{k}^{\beta} \hat{l}_{j}^{\beta}-\delta_{j k}\right)+\left[\frac{W^{\prime \prime}(\theta)}{s^{2} l_{\alpha} l_{\beta}}-\frac{\hat{l}_{m}^{1} \hat{l}_{m}^{2}}{l_{\alpha} l_{\beta}} \frac{W^{\prime}(\theta)}{s^{3}}\right]\left(\hat{l}_{k}^{\alpha} \hat{l}_{i}^{\alpha}-\delta_{i k}\right)\left(\hat{l}_{n}^{\beta} \hat{l}_{j}^{\beta}-\delta_{j n}\right) \hat{l}_{k}^{\beta} \hat{l}_{n}^{\alpha} .
$$

Otherwise, if $\alpha=\beta$ represents an exterior node and $\gamma$ denotes the other exterior node, we obtain

$$
K_{i j}^{\alpha \alpha}=-\frac{\hat{l}_{j}^{\alpha}}{l_{\alpha}} F_{i}^{\alpha}+\frac{1}{l_{\alpha}}\left[\left(\hat{l}_{k}^{\alpha} \hat{l}_{i}^{\alpha}-\delta_{i k}\right) \hat{l}_{k}^{\gamma}\left(\frac{W^{\prime \prime}(\theta)}{s} \frac{\partial \theta}{\partial u_{j}^{\alpha}}-\frac{W^{\prime}(\theta)}{s^{2}} \frac{\partial s}{\partial u_{j}^{\alpha}}\right)-\frac{W^{\prime}(\theta)}{s l_{\alpha}} \hat{l}_{k}^{\gamma}\left(\hat{l}_{k}^{\alpha}\left(\hat{l}_{i}^{\alpha} \hat{l}_{j}^{\alpha}-\delta_{i j}\right)+\hat{l}_{i}^{\alpha}\left(\hat{l}_{k}^{\alpha} \hat{l}_{j}^{\alpha}-\delta_{j k}\right)\right)\right]
$$

where for all exterior nodes $\alpha$

$$
\frac{\partial s}{\partial u_{i}^{\alpha}}=\hat{l}_{1} \cdot \hat{\boldsymbol{l}}_{2} \frac{\partial \theta}{\partial u_{i}^{\alpha}}, \quad \frac{\partial \theta}{\partial u_{i}^{\alpha}}=\frac{1}{s l_{\alpha}}\left[\hat{l}_{\alpha} \cdot \hat{\boldsymbol{l}}_{\gamma} \hat{l}_{i}^{\alpha}-\hat{l}_{i}^{\gamma}\right] .
$$

Next, if $\alpha$ is an exterior node and 0 refers to the central node (and, again, $\gamma$ being the other exterior node), then

$$
K_{i j}^{\alpha 0}=\frac{\hat{l}_{j}^{\alpha}}{l_{\alpha}} F_{i}^{\alpha}+\frac{1}{l_{\alpha}} D_{j k}^{\alpha}\left(\hat{l}_{k}^{\alpha} \hat{l}_{i}^{\alpha}-\delta_{i k}\right)+\frac{W^{\prime}(\theta)}{s l_{\alpha}^{2}} \hat{l}_{k}^{\gamma}\left(2 \hat{l}_{k}^{\alpha} \hat{l}_{j}^{\alpha} \hat{l}_{i}^{\alpha}-\delta_{i j} \hat{l}_{k}^{\alpha}-\delta_{j k} \hat{l}_{i}^{\alpha}\right)
$$

with

and

$$
D_{i j}^{\alpha}=\frac{W^{\prime \prime}(\theta)}{s} \hat{l}_{i}^{\gamma} \frac{\partial \theta}{\partial u_{j}^{0}}+\frac{W^{\prime}(\theta)}{s l_{\gamma}}\left(\hat{l}_{i}^{\gamma} \hat{l}_{j}^{\gamma}-\delta_{i j}\right)-\frac{W^{\prime}(\theta)}{s^{2}} \hat{l}_{i}^{\gamma} \frac{\partial s}{\partial u_{j}^{0}}
$$

$$
\frac{\partial s}{\partial u_{i}^{0}}=\hat{\boldsymbol{l}}_{1} \cdot \hat{\boldsymbol{l}}_{2} \frac{\partial \theta}{\partial u_{i}^{0}}, \quad \frac{\partial \theta}{\partial u_{j}^{0}}=-\frac{1}{s}\left(\frac{\hat{l}_{k}^{\gamma}}{l_{\alpha}}\left(\hat{l}_{k}^{\alpha} \hat{l}_{j}^{\alpha}-\delta_{j k}\right)+\frac{\hat{l}_{k}^{\alpha}}{l_{\gamma}}\left(\hat{l}_{k}^{\gamma} \hat{l}_{j}^{\gamma}-\delta_{j k}\right)\right) .
$$


Note that symmetry of the stiffness matrix requires that $K_{i j}^{\alpha 0}=K_{j i}^{0 \alpha}$.

Finally, the matrix components associated with $\alpha=\beta=0$ (force on the central node due to displacements of the central node) are computed as

$$
K_{i j}^{00}=\sum_{k=1}^{2}\left[\frac{W^{\prime \prime}(\theta)}{s} \frac{\partial \theta}{\partial u_{j}^{0}} G_{i k}-\frac{W^{\prime}(\theta)}{s^{2}} \frac{\partial s}{\partial u_{j}^{0}} G_{i k}+\frac{W^{\prime}(\theta)}{s} \frac{\partial G_{i k}}{\partial u_{j}^{0}}\right]
$$

where we defined

$$
G_{i k}=-\frac{\hat{l}_{k}^{1}}{l_{2}}\left(\hat{l}_{k}^{2} \hat{l}_{i}^{2}-\delta_{i k}\right)-\frac{\hat{l}_{k}^{2}}{l_{1}}\left(\hat{l}_{k}^{1} \hat{l}_{i}^{1}-\delta_{i k}\right) .
$$

Computing and simplifying all partial derivatives results in

$$
\begin{aligned}
\frac{\partial G_{i k}}{\partial u_{j}^{0}}= & -\frac{1}{l_{1} l_{2}}\left(\hat{l}_{k}^{1} \hat{l}_{j}^{1}-\delta_{j k}\right)\left(\hat{l}_{k}^{2} \hat{l}_{i}^{2}-\delta_{i k}\right)-\frac{1}{l_{1} l_{2}}\left(\hat{l}_{k}^{2} \hat{l}_{j}^{2}-\delta_{j k}\right)\left(\hat{l}_{k}^{1} \hat{l}_{i}^{1}-\delta_{i k}\right)-\frac{1}{l_{2}^{2}} \hat{l}_{j}^{2} \hat{l}_{k}^{1}\left(\hat{l}_{k}^{2} \hat{l}_{i}^{2}-\delta_{i k}\right)-\frac{1}{l_{1}^{2}} \hat{l}_{j}^{1} \hat{l}_{k}^{2}\left(\hat{l}_{k}^{1} \hat{l}_{i}^{1}-\delta_{i k}\right) \\
& -\frac{\hat{l}_{k}^{1}}{l_{2}^{2}}\left(2 \hat{l}_{k}^{2} \hat{l}_{j}^{2} \hat{l}_{i}^{2}-\delta_{i j} \hat{l}_{k}^{2}-\delta_{j k} \hat{l}_{i}^{2}\right)-\frac{\hat{l}_{k}^{2}}{l_{1}^{2}}\left(2 \hat{l}_{k}^{1} \hat{l}_{j}^{1} \hat{l}_{i}^{1}-\delta_{i j} \hat{l}_{k}^{1}-\delta_{j k} \hat{l}_{i}^{1}\right) .
\end{aligned}
$$

